\title{
WEAK MAPS OF COMBINATORIAL GEOMETRIES
}

\author{
BY
}

\section{DEAN LUCAS}

\begin{abstract}
Weak maps of combinatorial geometries are studied, with particular emphasis on rank preserving weak bijections. Equivalent conditions for maps to be reversed under duality are given. It is shown that each simple image (on the same rank) of a binary geometry $G$ is of the form $G / F \oplus F$ for some subgeometry $F$ of $G$. The behavior of invariants under mappings is studied. The Tutte polynomial, Whitney numbers of both kinds, and the Möbius function are shown to behave systematically under rank preserving weak maps. A weak map lattice is presented and, through it, the lattices of elementary images and preimages of a fixed geometry are studied.
\end{abstract}

1. Introduction. In the field of combinatorial geometry strong maps have been widely studied ([7], [8], [11], [12]). This paper examines the related concept of weak maps [7]. Weak maps are generalizations of strong maps. The weak maps which preserve rank are, however, "orthogonal" to strong maps in the sense of $\S 4$ and are singled out for special study in later sections.

In $\S 4$ we show that every weak map has a decomposition into an alternating sequence of rank preserving weak maps and truncations. We also give necessary and sufficient conditions for maps to be reversed under duality.

$\S \S 5,6$, and 7 deal with properties preserved by weak images and preimages. $\$ 5$ describes the action of rank preserving weak maps on circuits, flats, and minors. $\$ 7$ shows how rank preserving weak maps affect the Tutte polynomial of a geometry and various derived invariants, such as the Möbius function and the Crapo invariant. $\S 6$ discusses maps of binary geometries. These turn out to have a particularly simple form. Indeed, any simple (i.e. minimal) rank preserving image of a binary geometry $G$ is of the form $G / F \oplus F$ for some subgeometry $F$ of $G$.

In $\S 8$ weak and strong maps are related by viewing the lattice of modular cuts in a weak map context. Weak map extensions are discussed and the relation of the weak map partial order to the Higgs lattice order is described.

Received by the editors February 5, 1974.

AMS (MOS) subject classifications (1970). Primary 05B25, 05B35; Secondary 05A20, 15A03, 15A33, 50D30.

Key words and phrases. Combinatorial geometry, combinatorial pregeometry, weak map, strong map, simple map, binary geometry, Tutte polynomial, matroid, Whitney numbers. 
2. Basic concepts. The material in this section is widely known and is therefore stated here without proof. A reader who would like a detailed exposition of these topics should consult [7] or [2].

A combinatorial pregeometry $G$ is a finite set $X$, whose elements are called points, together with a family $I_{G}$ of subsets of $X$ which are called the independent sets of $G$ and satisfy these axioms:

(1) $\varnothing \in I_{G}$.

(2) If $I \in I_{G}$ and $I^{\prime} \subseteq I$ then $I^{\prime} \in I_{G}$.

(3) If $I_{1}$ and $I_{2}$ are in $I_{G}$ and $\left|I_{1}\right|=\left|I_{2}\right|-1$ then there is a point $p \in I_{2}$ such that $I_{1} \cup p \in I_{G}$.

Maximal independent sets of $G$ are called bases. $B_{G}$ will represent the set of bases for $G$. Dependent sets of $G$ are those subsets of $X$ not in $I_{G}$. Minimal dependent sets are called circuits. $C_{G}$ will represent the set of circuits of $G$. The rank in $G$ of a subset $A$ of $X$, written $r_{G}(A)$, is the size of a maximal independent subset of $A$. A subset $I$ of $X$ is in $I_{G}$ iff $r_{G}(I)=|I|$. The $G$-closure of a subset $A$ of $X$, written $\mathrm{Cl}_{G}(A)$, is the set of all points $p$ of $X$ such that $r_{G}(A \cup p)=r_{G}(A)$. The flats of $G$ are the subsets $f$ of $X$ such that $\mathrm{Cl}_{G}(f)=f$. Flats are maximal subsets of a given rank. The hyperplanes of $G$ are those flats whose rank is $r_{G}(X)-1$. Each flat is equal to the intersection of all hyperplanes that contain it. The flats and hyperplanes of $G$ will be denoted by $F_{G}$ and $H_{G}$, respectively. The flats of $G$ form a lattice $L_{G}$ when ordered by containment. The infimum of two flats in $L_{G}$ is their intersection.

A pregeometry $G$ on a set $X$ is called a geometry if every two element subset of $X$ is independent in $G$, or, equivalently, if $G$ has no circuit $C$ with $|C|=1$ or $|C|=2$.

Throughout this paper we will drop the cumbersome and somewhat demeaning prefix "pre" and refer to pregeometries simply as geometries. On the rare occasions when we wish to specify a geometry in the strict sense we will use the term "proper geometry". Also we will not distinguish between a geomtry and its underlying set if the meaning is clear. For instance we will use $|G|=n$ to mean that $G$ is a geometry on a set of size $n$. We will write $r(G)$ instead of $r_{G}(X)$ and refer to this number simply as the rank of $G$. The reader will already have noticed that we express the union of a set $A$ and a singleton set $\{p\}$ as $A \cup p$ instead of the clumsy $A \cup\{p\}$.

Given a geometry $G$ on the set $X$ with basis set $B_{G}$ then the set $B_{G}^{*}=$ $\left\{X-B \mid B \in B_{G}\right\}$ satisfies the basis axioms and therefore defines another geometry $G^{*}$ on $X$ which is called the (Whitney) dual of $G$. Thus $B_{G^{*}}=B_{G}^{*}$.

From the definition, bases of $G^{*}$ are complements of bases of $G$. It follows that circuits of $G^{*}$ are complements of hyperplanes of $G$ and hyperplanes 
of $G^{*}$ are complements of circuits of $G$. The rank function on $G^{*}$ is $r_{G^{*}}(A)=$ $|A|-r(G)+r_{G}(X-A)$. In particular $r\left(G^{*}\right)=|X|-r(G)$. Also $\left(G^{*}\right)^{*}=G$.

Given a geometry $G$ on $X$ and $p \in X$, the deletion from $G$ of $p, G-p$, is the geometry on $X-p$ with independent sets: $I_{G-p}=\left\{I \in I_{G} \mid p \notin I\right\}$.

The contraction of $G$ by $p, G / p$, is the geometry on $X-p$ whose independent sets are given by $I_{G / p}=\left\{I \in I_{G} \mid p \notin I\right.$ and $\left.I \cup p \in I_{G}\right\}$ provided $p$ is not a circuit of $G$, and $I_{G / p}=I_{G-p}$ if $p$ is a circuit of $G$. Let $S$ be an arbitrary sequence of deletions and contractions of points of $X$. For instance, $S$ could be of the form $S=\left(/ p_{1},-p_{2},-p_{3}, / p_{4}, \cdots,-p_{m}\right)$. If the sequence of operations $S$ is applied to $G$ the resulting geometry $M$ is called a minor of $G$. It is known [1] that the application of any permutation $S^{\prime}$ of $S$ to $G$ results in the same minor $M$. Thus if $A$ is the set of points to be contracted in the sequence $S$ and $B$ is the set of points to be deleted in $S$ we may express the minor $M$ unambiguously as $M=G / A-B$. The independent sets of $M$ are as follows: $I_{M}=$ $\left\{I \subset X-(A \cup B) \mid I \cup I^{\prime} \in I_{G}\right.$ for all bases $I^{\prime}$ of $\left.A\right\}$. A minor of the form $G-$ $B$ (no contractions) is called a subgeometry of $G$. Using our convention of not distinguishing between a geometry and its underlying set, a subgeometry $F$ of $G$ can be expressed as $F=G-(X-F)$. A minor of the form $G / A$ is called the contraction of $G$ by $A$.

Given geometries $G_{1}$ on $X_{1}$ and $G_{2}$ on $X_{2}$ we form a new geometry $G_{1} \oplus G_{2}$ on $X_{1} \cup X_{2}$ whose independent sets are $I_{G_{1} \oplus G_{2}}=\left\{I_{1} \cup I_{2} I_{1} \in I_{G_{1}}\right.$, $I_{2} \in I_{G_{2}}$ \}. $G_{1} \oplus G_{2}$ is called the direct sum of $G_{1}$ and $G_{2}$. If a geometry $G$ can be expressed as the direct sum of two nonempty geometries $G_{1}$ and $G_{2}$ then $G$ is called separable and $G_{1}$ and $G_{2}$ are called separators of $G$. Equivalently, $G_{1}$ and $G_{2}$ are separators of $G$ if no circuit of $G$ has nonempty intersection with both $G_{1}$ and $G_{2}$. A nonseparable geometry is called connected. A separator of $G$ which is itself connected is called a component of $G$.

A component of $G$ which consists of a single point $p$ may be of two types. Regarded as a subgeometry of $G, p$ may be either independent or dependent. In the former case $p$ is called an isthmus and in the latter it is called a loop. If we wish to indicate that a one point separator is a loop we will place it in parentheses. Thus for any geometry $H, p$ is an isthmus of $H \oplus p$ and $p$ is a loop of $H \oplus(p)$.

Duals, minors, and separators interact as follows:

$2.1(G / A-B)^{*}=G^{*} / B-A$, in particular $(G-p)^{*}=G^{*} / p$ and $(G / p)^{*}=$ $G^{*}-p$.

$2.2\left(G_{1} \oplus G_{2}\right)^{*}=G_{1}^{*} \oplus G_{2}^{*}$.

$2.3\left(G_{1} \oplus G_{2}\right) / A-B=\left[G_{1} /\left(A \cap X_{1}\right)-\left(B \cap X_{1}\right)\right] \oplus\left[G_{2} /\left(A \cap X_{2}\right)-\right.$ $\left.\left(B \cap X_{2}\right)\right]$. 
Given a set $X$ with $|X|=n$ we wish to single out certain distinguished geometries on $X$. The unique geometry whose bases consist of all the $k$-subsets of $X$ (for some $k$ with $0 \leqslant k \leqslant n$ ) is called the free geometry of rank $k$ on $X$ and written $F_{X, k}$. The free geometry of rank $n$ on $X$ is called the Boolean algebra on $X$ and written $B_{X}$. Every point of $B_{X}$ is an isthmus. A geometry having $k$ isthmuses and the remaining $n-k$ points loops is called a pre-Boolean algebra of rank $k$ on $X$.

Certain abstract combinatorial geometries can be represented by more concrete objects. Our preference in this paper will be to represent geometries as subsets of points in affine space whenever possible. In an affine representation loops will be indicated by placing points in parentheses. Several points touching one another will indicate that those points form a flat of rank 1, or multiple point. Flats of higher rank are shown as lines, planes, etc. In the affine picture flats are not drawn unless they are dependent. For instance a three point line is drawn but a two point line is not.

3. The weak map partial order. In this section we define and discuss two order relations, one on the set of all geometries on a set $X$ and the other on the set of isomorphism classes of $n$-point geometries.

DEFintion 3.1. Let $P_{X}$ be the set of all (pre) geometries on a finite set $X$. For $G$ and $H$ in $P_{X}$ we say that the identity map from $G$ to $H$ is weak, written $G \rightarrow H$, provided $I_{G} \supseteq I_{H}$. The proofs of the following two propositions are straightforward.

Proposition 3.2. For $G$ and $H$ in $P_{X}$ the following are equivalent:

(1) $G \rightarrow H$.

(2) Each independent set of $H$ is independent in $G$.

(3) Each dependent set of $G$ is dependent in $H$.

(4) Each G-circuit (circuit of $G$ ) contains an H-circuit.

(5) $r_{G}(A) \geqslant r_{H}(A)$ for all $A \subseteq X$.

PROPOSITION 3.3. The relation " $\rightarrow$ " is a partial order on $P_{X}$ which we will call the weak map partial order. This partial order has greatest element $B_{X}$ and least element $B_{X}^{*}$. When restricted to proper geometries the partial order has least element $F_{X, 2}$.

DEFINITION 3.4. Two geometries $G$ on $X$ and $G^{\prime}$ on $X^{\prime}$ are isomorphic if there exists a bijection $\beta: X \rightarrow X^{\prime}$ such that $I \in I_{G}$ iff $\beta(I) \in I_{G^{\prime}}$. The isomorphism class of a geometry $G$ is the collection of all geometries isomorphic to $G$.

Let $P_{n}$ be the set of all isomorphism classes of $n$ point (not necessarily 
proper) geometries. For reasons that will soon become apparent we will use virtually the same notation when discussing $P_{n}$ as we use for $P_{X}$. Whereas $G \in$ $P_{X}$ means that $G$ is a geometry on $X, H \in P_{n}$ means that $H$ is an isomorphism class of $n$ point geometries. By the statement $p \in G$ for $G \in P_{n}$, we will mean that given a representative $G^{\prime}$ of $G$ then $p$ represents a point $p^{\prime}$ of $G^{\prime}$ and given another representative $G^{\prime \prime}$ isomorphic to $G^{\prime}$ by $\beta: G^{\prime} \rightarrow G^{\prime \prime}$ then $p$ represents the point $\beta\left(p^{\prime}\right)$ of $G^{\prime \prime}$. Using this definition of $p \in G$ we may introduce the well-defined isomorphism classes $G-p$ and $G / p$, the isomorphism classes of $G^{\prime}-p^{\prime}$ and $G^{\prime} / p^{\prime}$ respectively.

We will change notation slightly to indicate the isomorphism classes of the distinguished geometries of $\S 2$. We write $F_{n, k}$ for the isomorphism class of $F_{X, k}, B_{n}$ for the isomorphism class of $B_{X}$, and $B_{n, k}$ for the isomorphism class containing the pre-Boolean algebras on $X$ of rank $k$. Thus $F_{n, n}=B_{n}=B_{n, n}$.

Definition 3.5. For $G$ and $H$ in $P_{n}$ we say there is a weak map from $G$ to $H$, written $G \rightarrow H$, if for any representatives $G^{\prime}$ of $G$ and $H^{\prime}$ of $H$ there is a bijection $b$ on the underlying sets such that whenever $I$ is an independent subset of $H^{\prime}$ then $b^{-1}(I)$ is independent in $G^{\prime}$. A bijection with this property is called a weak bijection.

PROPOSITION 3.6. For $G$ and $H$ in $P_{n}$ the following are equivalent:

(1) $G \rightarrow H$.

(2) There exist representatives $G^{\prime}$ of $G$ and $H^{\prime}$ of $H$ with a weak bijection b: $G^{\prime} \rightarrow H^{\prime}$.

(3) For any $n$-set $X$ there exist representatives $G$ " of $G$ and $H^{\prime \prime}$ of $H$ both on $X$ such that $G^{\prime \prime} \rightarrow H^{\prime \prime}$ in $P_{X}$.

Proof. This follows immediately by composing isomorphisms and weak bijections.

Throughout the remainder of this paper we will make many statements about weak maps in $P_{X}$ or $P_{n}$. Our general tactic will be to prove statements for weak maps in $P_{X}$ only. The proof in $P_{X}$ applied to $G^{\prime \prime}$ and $H^{\prime \prime}$ of (3.6) gives the proof for $P_{n}$.

PROPOSITION 3.7. The relation " $\rightarrow$ " is a partial order on $P_{n}$ which we will call the weak map partial order. This partial order has greatest element $B_{n}$ and least element $B_{n}^{*}$.

PRoof. This partial order is obtained from the weak map partial order on $P_{X}$ by identifying isomorphic geometries. One checks easily that the partial order is preserved in the quotient.

Defintion 3.8. Let $G$ and $H$ be in $P_{X}$ or $P_{n}$. A weak map $G \rightarrow H$ will 
be referred to simply as a map unless we wish to emphasize that it has no stronger qualities. A map $G \rightarrow H$ is called trivial if $G=H$, nontrivial otherwise. A map $G \rightarrow H$ is called simple if $G$ covers $H$ in the relevant partial order, i.e. if for all $F$ for which there are maps $G \rightarrow F \rightarrow H$, exactly one of the maps $G \rightarrow F$ and $F \rightarrow H$ is trivial. It is clear that any map $G \rightarrow H$ has a decomposition into simple maps: $G \rightarrow F_{1} \rightarrow F_{2} \cdots F_{m} \rightarrow H$.

Proposition 3.9. Let $P_{X, k}$ represent the set of all geometries on $X$ of rank $k$ and let $P_{n, k}$ represent the set of all isomorphism classes of $n$ point geometries of rank $k$. $P_{X, k}$ and $P_{n, k}$ are convex sets in the weak map partial order on $P_{X}$ and $P_{n}$ respectively. $P_{X, k}$ has $F_{X, k}$ as its unique maximal element and its minimal elements are the $\left(\begin{array}{c}n \\ k\end{array}\right)$ pre-Boolean algebras on $X . P_{n, k}$ is the interval $\left[F_{n, k}, B_{n, k}\right]$ of $P_{n}$.

Proof. That $P_{X, k}$ and $P_{n, k}$ are convex sets is clear from the fact that weak maps cannot raise rank. Any $G \in P_{X, k}$ has no independent sets of size greater than $k$ so we have the map $F_{X, k} \rightarrow G$. Since $r(G)=k, G$ has a basis $B$ of size $k$. Let $B^{\prime}$ be the pre-Boolean algebra which has the points in $B$ as isthmuses and all other points loops. Then $r\left(B^{\prime}\right)=k$ and we have the map $G \rightarrow B^{\prime}$. The same argument applied to a representative $G^{\prime \prime}$ of $G \in P_{n, k}$ shows that there are maps $F_{n, k} \rightarrow G$ and $G \rightarrow B_{n, k}$.

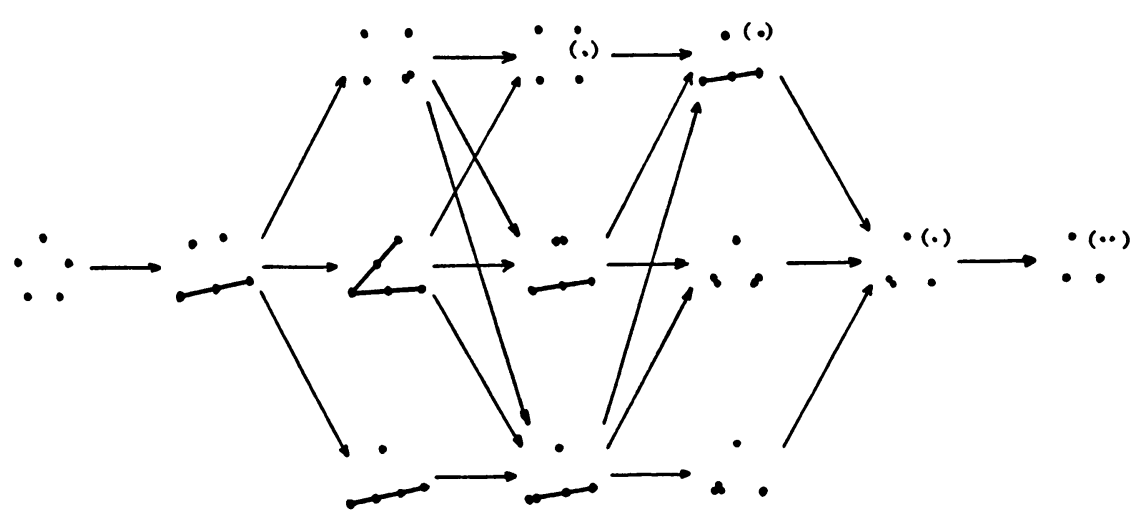

FIGURE 3.10. The weak map partial order on $P_{5,3}$ with simple maps shown

The weak map partial order on $P_{X}, P_{n}, P_{X, k}$, and $P_{n, k}$ is not, in general, a lattice nor does it satisfy the Jordan-Dedekind chain condition. The nonexistence of suprema and infima is shown in Figure 3.10. Figure 8.4 shows the failure of the chain condition. 
4. Some special types of maps. We will need to distinguish two important classes of weak maps.

DEFINTION 4.1. For $G$ and $H$ in $P_{X}$ or $P_{n}$ we call a weak map $G \rightarrow H$ rank preserving if $r(G)=r(H)$. Rank preserving weak maps will be referred to as rp maps and written as $G \stackrel{\mathrm{rp}}{\longrightarrow} H$.

The next type of map has been widely studied ([7], [8], [11], [12]).

DEFintion 4.2. For $G$ and $H$ in $P_{X}$ or $P_{n}$ we say there is a strong map from $G$ to $H$, written $G \stackrel{\text { st }}{\longrightarrow} H$, provided each closed set in $H$ is closed in $G$ (this definition applies to $P_{n}$ via (3.6)). Equivalently, $G \rightarrow H$ is strong iff each $G$-circuit is a union of $H$-circuits. A strong map $G \rightarrow H$ is called elementary if $r(G)-r(H)=1$. It follows from the first form of the definition that a composition of strong maps is strong. From the second form of the definition it is clear that strong maps are weak. Higgs [11] has shown that any strong map $G \stackrel{\text { st }}{\longrightarrow} H$ has a decomposition into elementary strong maps: $G \stackrel{\text { st }}{\longrightarrow} F_{1} \stackrel{\text { st }}{\longrightarrow} F_{2}$ $\cdots F_{m} \stackrel{\text { st }}{\longrightarrow} H$.

The next three propositions show that these two classes of maps behave in a manner which is, loosely speaking, orthogonal.

PROPOSITION 4.3. Rank preserving maps are preserved under duality. Thus $G \stackrel{\mathrm{rp}}{\longrightarrow} H$ iff $G^{*} \stackrel{\mathrm{rp}}{\longrightarrow} H^{*}$. If the former map is simple then so is the latter.

Proof. Since $r(G)=r(H)$ every basis of $H$ is a basis of $G$. Thus every basis complement of $H$ is a basis complement of $G$. But these basis complements are exactly the bases of the dual geometries so $G^{*} \stackrel{\mathrm{rp}}{\longrightarrow} H^{*}$.

COROLlary 4.4. The function sending $G$ to $G^{*}$ is an order isomorphism between $P_{x, k}$ and $P_{x, n-k}$ and between $P_{n, k}$ and $P_{n, n-k}$, under the weak map partial order.

The first statement in the next proposition appears in [1]

Proposition 4.5. Strong maps are reversed under duality. Thus $G \stackrel{\text { st }}{\longrightarrow} H$ iff $H^{*} \stackrel{\text { st }}{\longrightarrow} G^{*}$. One of the above maps can be simple and the other not simple. The dual of an elementary map is, however, elementary.

Proof. The second statement is illustrated by this example.

The map $\stackrel{a}{b} \stackrel{\text { st }}{\longrightarrow}{ }^{a} \infty^{b}$ is simple but the dual map decomposes as shown:

$$
[\bullet a \bullet b]^{*}=\left((a \bullet b) \longleftarrow a \bullet b=\left[\begin{array}{lll}
a \bullet b & *
\end{array}\right]^{*}\right.
$$


The last sentence of the proposition follows from the fact that if $r(G)=r(H)+$ 1 , then $r\left(H^{*}\right)=n-r(H)=n-(r(G)-1)=n-r(G)+1=r\left(G^{*}\right)+1$.

The next proposition appears in [7]. The proof given here is new.

PROPOSITION 4.6. A map that is both rank preserving and strong is trivial.

Proof. Suppose $G \rightarrow H$ is rank preserving and strong. By 4.3 we have $G^{*} \rightarrow H^{*}$ and by 4.5 we have $H^{*} \rightarrow G^{*}$, so the antisymmetry of the partial order gives $G^{*}=H^{*}$. Therefore $G=H$.

Definition 4.7. For $G$ in $P_{X}$ with $r(G)>0$ the truncation of $G, T(G)$, is the geometry whose independent sets are: $I_{T(G)}=\left\{I \in I_{G}|| I \mid \leqslant r(G)-1\right\}$. Equivalently the flats of $r(G)$ are: $\left\{f \subseteq X \mid f\right.$ is a $G$-flat and $\left.r_{G}(f) \neq r(G)-1\right\}$. If $G$ has rank 0 we define $T(G)=G$. For $G$ in $P_{n}, T(G)$ represents the unique equivalence class containing the truncations of all representations of $G$. Thus $T$ is a function from $P_{X}$ to $P_{X}$ or $P_{n}$ to $P_{n} . T^{k}(G)$ will mean the truncation of $G k$ times.

It is clear from the second form of the definition that $G \rightarrow T(G)$ is a strong map and is elementary if $r(G) \neq 0$.

The next proposition collects some useful properties of truncations.

Proposition 4.8. Let $G$ and $H$ be in $P_{X}$ or $P_{n}$.

(a) $G \rightarrow H$ iff $T^{r(G)-r(H)}(G) \stackrel{\text { rp }}{\longrightarrow} H$.

(b) If $G \rightarrow H$ then $T(G) \rightarrow T(H)$.

(c) Every simple map which reduces rank is a truncation.

Proof. (a) Each independent set $I$ of $H$ is independent in $G$ and has size $|I| \leqslant r(H)=r(G)-(r(G)-r(H))$. Therefore $I$ is still independent in $T^{r(G)-r(H)}(G)$. Conversely if $T^{r(G)-r(H)}(G) \stackrel{\mathrm{ID}}{\longrightarrow} H$ then compose that map with $G \stackrel{\text { st }}{\longrightarrow} T^{r(G)-r(H)}(G)$ to get $G \rightarrow H$. $T(H)$.

(b) Apply (a) to the composite map $G \rightarrow H \stackrel{\text { st }}{\longrightarrow} T(H)$ to get $T(G) \rightarrow$

(c) Suppose $G \rightarrow H$ is a simple rank reducing map. Using (a) we have $G \stackrel{\text { st }}{\longrightarrow} T(G) \stackrel{\text { st }}{\longrightarrow} T^{r(G)-r(H)}(G) \stackrel{\text { rp }}{\longrightarrow} H$. The first of these maps is nontrivial so both the others must be trivial, and $T(G)=H$.

Definition 4.9. For $G$ in $P_{X}$ the lift of $G, L(G)$, is defined by $L(G)=$ $\left(T\left(G^{*}\right)\right)^{*}$ (this is the Higgs lift of the map $B_{X} \stackrel{\text { st }}{\longrightarrow} G$ as defined in [11] or [12]). For $G$ in $P_{n}, L(G)$ is the unique isomorphism class containing all lifts of representatives of $G$. Thus $L$ is a function from $P_{X}$ to $P_{X}$ or $P_{n}$ to $P_{n}$. $L^{k}(G)$ will denote the result of applying a $L$ to $G k$ times.

The next proposition collects some useful properties of $L$. 
Proposition 4.10. Let $G$ be in $P_{X}$ or $P_{n}$.

(a) $L(G) \rightarrow G$ is a strong map and is elementary if $r(G) \neq n$.

(b) Given a strong map $H \stackrel{\text { st }}{\longrightarrow} G$ with $r(H)-r(G)=k$ then $L^{k}(G) \stackrel{\mathrm{rp}}{\longrightarrow} H$.

(c) If $H \stackrel{\mathrm{rp}}{\longrightarrow} G$ then $L(H) \stackrel{\mathrm{rp}}{\longrightarrow} L(G)$.

Proof. Part (a) follows from the fact that $L(G) \rightarrow G$ is the dual of the truncation map $G^{*} \stackrel{\text { st }}{\longrightarrow} T\left(G^{*}\right)$. For (b) suppose $H \stackrel{\text { st }}{\longrightarrow} G$ with $r(H)-r(G)=k$. Then $G^{*} \stackrel{\text { st }}{\longrightarrow} H^{*}$ and $r\left(G^{*}\right)-r\left(H^{*}\right)=k$ so from $4.8 T^{k}\left(G^{*}\right) \stackrel{\text { rp }}{\longrightarrow} H^{*}$. We redualize to get $\left(T^{k}\left(G^{*}\right)\right)^{*} \stackrel{\mathrm{rp}}{\longrightarrow} H$. But $\left(T^{k}\left(G^{*}\right)\right)^{*}=L^{k}(G)$ since the internal duals cancel in pairs. Therefore $L^{k}(G) \stackrel{\mathrm{rp}}{\longrightarrow} H$, as required. For (c) let $H \stackrel{\mathrm{rp}}{\longrightarrow}$ G. Then $H^{*} \stackrel{\mathrm{rp}}{\longrightarrow} G^{*}$ so by $4.8 T\left(H^{*}\right) \stackrel{\mathrm{rp}}{\longrightarrow} T\left(G^{*}\right)$ and therefore $L(H) \stackrel{\mathrm{rp}}{\longrightarrow} L(G)$.

Propositions 4.3 and 4.5 described the behavior of rp maps and strong maps under duality. We close this section by discussing the behavior under duality of arbitrary weak maps.

DEFINITION 4.11. A map $G \rightarrow H$ extends to a strong map on the same rank provided there are rp maps $G_{1} \stackrel{\mathrm{rp}}{\longrightarrow} G$ amd $H \stackrel{\mathrm{rp}}{\longrightarrow} H_{1}$ such that the composite map $G_{1} \stackrel{\mathrm{rp}}{\longrightarrow} G \longrightarrow H \stackrel{\mathrm{rp}}{\longrightarrow} H_{1}$ from $G_{1}$ to $H_{1}$ is strong.

THEOREM 4.12. For a weak map $G \rightarrow H$ with $r(G)-r(H)=k$ the following are equivalent:

(a) $G \rightarrow H$ extends to a strong map on the same rank.

(b) $H^{*} \rightarrow G^{*}$.

(c) $L^{k}(H) \stackrel{\mathrm{rp}}{\longrightarrow} G$.

Proof. If (a) holds we have

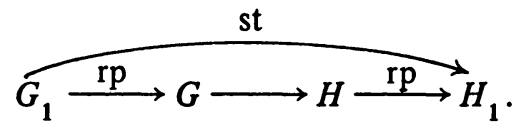

Dualizing gives $H^{*} \stackrel{\mathrm{rp}}{\longrightarrow} H_{1}^{*} \stackrel{\text { st }}{\longrightarrow} G_{1}^{*} \stackrel{\mathrm{rp}}{\longrightarrow} G^{*}$, so $H^{*} \rightarrow G^{*}$ as required. If $H^{*} \rightarrow G^{*}$ then by $4.8 T^{k}\left(H^{*}\right) \stackrel{\mathrm{rp}}{\longrightarrow} G^{*}$. Dualizing gives $\left(T^{k}\left(H^{*}\right)\right)^{*}=L^{k}(H)$ $\stackrel{\mathrm{rp}}{\longrightarrow} G$. If $L^{k}(H) \stackrel{\mathrm{rp}}{\longrightarrow} G$ then the composite $L^{k}(H) \rightarrow H$ is a strong map which extends $G \rightarrow H$ on the same rank.

5. Hereditary properties: general. In this section and the two that follow we will be concerned with properties that are preserved by weak maps and weak map inverses. Some of the results presented in these sections were stated without proof in [13].

Proposition 5.1. For $G$ and $H$ in $P_{X}$ or $P_{n}$ if $G \rightarrow H$ and $H$ is proper geometry or isomorphism class of proper geometries then so is $G$.

Proof. If $G$ is not proper it has a 1 or 2 point circuit. A 1 point 
$G$-circuit must contain an $H$-circuit and hence must be a 1 point $H$-circuit. A 2 point $G$-circuit must either remain a circuit in $H$ or contain a 1 point $H$-circuit. In either case $H$ is not a proper geometry.

Little else can be said about hereditary properties of arbitrary maps. We know from the previous sections, however, that any map has a decomposition into simple maps and that these simple maps fall into two classes: rp maps and truncations. We have also seen that these two types of maps exhibit quite different behavior, at least with respect to duality. Thus, in studying hereditary properties it might be wise to consider these two types of maps separately. Since truncations are well understood, our approach will be to study the hereditary properties of rp maps and mention, in passing, how these properties relate to truncations.

Proposition 5.2. For $G$ and $H$ in $P_{X}$, if there is an $\operatorname{rp~map~} G \stackrel{\mathrm{rp}}{\longrightarrow} H$ and $S$ is a separator of $G$ then the subgeometry $S^{\prime}=H-(X-S)$ of $H$ is a separator of $H$.

Proof. If $S^{\prime}$ is not a separator of $H$ then there is an $H$-circuit $C$ with $p \in C \cap S^{\prime}$ and $q \in C \cap\left(X-S^{\prime}\right)$. We extend the independent set $C-p$ to a basis $B$ of $H$. $B$ must also be a basis of $G$. The point $p$ makes a unique $G$-circuit $C_{1}$ with $B$. Now $C_{1}$ must contain an $H$-circuit $C_{2}$ and $C_{2}$ must contain $p$ (for $C_{1}-p \subseteq B$ ). If $C_{2}=C$ then $q \in C_{2}$ so $q \in C_{1}$, contradicting the fact that $S$ is a separator of $G$. If $C_{2} \neq C$ then there exists an $H$-circuit $C_{3} \subseteq\left(C_{2} \cup\right.$ $C)-p$. But this is impossible since $\left(C_{2} \cup C\right)-p \subseteq B$.

COROLlaRy 5.3. Rank preserving maps in $P_{X}$ or $P_{n}$ preserve separators.

Truncations, however, never preserve separators unless the separators in question are loops.

COROLLARY 5.4. Rank preserving maps send isthmuses to isthmuses, loops to loops.

Proof. A loop must remain a dependent separator and hence must remain a loop. The case for isthmuses follows by duality.

PROPOSITION 5.5. If $G$ and $H$ are in $P_{X}$ or $P_{n}$ and there is an rp map $G \stackrel{\mathrm{Ip}}{\longrightarrow} H$, then we have the following maps:

(a) $G-p \stackrel{\mathrm{rp}}{\longrightarrow} H-p$ if $p$ is not an isthmus of $H$.

(b) Dually, G/p $\stackrel{\mathrm{rp}}{\longrightarrow} H / p$ if $p$ is not a loop of $H$.

Proof. Since $I_{G} \supseteq I_{H}$ it is clear that $\left\{I \in I_{G} \mid p \notin I\right\} \supseteq\left\{I \in I_{H} \mid\right.$ $p \notin I\}$ so $G-p \rightarrow H-p$. Since $p$ is not an isthmus of $H$, by 5.4 it is not an isthmus of $G$. Thus $r(G-p)=r(G)=r(H)=r(H-p)$ so $G-p \stackrel{\mathrm{rp}}{\longrightarrow} H-p$. 
Definition 5.6. Let $M=G / A-B$ be a minor of a geometry $G$ where $A=\left\{p_{1}, p_{2}, \cdots, p_{m}\right\}$ and $B=\left\{p_{m+1}, p_{m+2}, \cdots, p_{e}\right\} . G / A-B$ is called a proper expression for $M$ if given any sequence $S$ of one point operations in which the points of $A$ are contracted and the points of $B$ deleted (i.e. $S=\left(/ p_{1}, / p_{2}\right.$, $\cdots, / p_{m},-p_{m+1}, \cdots,-p_{e}$ ) or some permutation thereof) then at no stage is a loop contracted or an isthmus deleted. If $G / A-B$ is a proper expression for $M$ then $M$ is said to be properly expressed by $G / A-B$.

Proposition 5.7. Any minor can be properly expressed.

Proof. Let $M=G / A^{\prime}-B^{\prime}$ be a minor of $G$ where $A^{\prime}=\left\{p_{1}, \cdots, p_{m}\right\}$ and $B^{\prime}=\left\{p_{m+1}, \cdots, p_{e}\right\}$. We know from $\S 2$ that $M$ can be expressed by any sequence of one point operations $S^{\prime}$ in which the points of $A^{\prime}$ are contracted and the points of $B^{\prime}$ are deleted. For convenience let $S^{\prime}=\left(/ p, \cdots, / p_{m}\right.$, $\left.-p_{m+1}, \cdots,-p_{e}\right)$. We begin applying the operations of $S^{\prime}$, in order, to $G$. At each step $/ p_{i}$ at which a loop is contracted we replace $/ p_{i}$ in the sequence by $-p_{i}$. At each step $-p_{j}$ at which an isthmus is deleted we replace $-p_{j}$ in the sequence by $/ p_{j}$. Call the resulting sequence of operations $S$. Applying $S$ to $G$ also results in the minor $M$ since deletion and contraction are identical operations if the point in question is a loop or an isthmus. Furthermore, we have constructed $S$ so that at no stage of the sequence is a loop contracted or an isthmus deleted.

A routine check shows that in no permutation of the operations of $S$ is a loop contracted or an isthmus deleted. Therefore letting $A=\left\{p_{i} \in A^{i} \cup B^{\prime} \mid p_{i}\right.$ is contracted in $S\}$ and $B=\left\{p_{j} \in A^{\prime} \cup B^{\prime} \mid p_{j}\right.$ is deleted $\left.S\right\}$ we have that $M=$ $G / A-B$ is a proper expression for the minor $M=G / A^{\prime}-B^{\prime}$.

THEOREM 5.8. Let $G$ and $H$ be in $P_{X}$ or $P_{n}$ with $G \stackrel{r p}{\longrightarrow} H$. Let $M$ be a minor of $H$ properly expressed by $M=H / A-B$ and let $N=G / A-B$ be the corresponding minor of $G$. Then there is an $\mathrm{rp} \operatorname{map} N \stackrel{\mathrm{rp}}{\longrightarrow} M$.

PROof. Express $M$ by applying the sequence of operations $S=\left(/ p_{1}, \cdots\right.$, $\left.\mid p_{m},-p_{m+1}, \cdots,-p_{e}\right)$ to $H$ where $A=\left\{p_{1}, \cdots, p_{m}\right\}$ and $B=\left\{p_{m+1}, \cdots\right.$, $p_{e}$ \}. Now apply the sequence $S$ to $G$, invoking 5.5 at each step, to obtain the desired result.

COROLlaRY 5.9. If there is an $\mathrm{rp}$ map $G \stackrel{\mathrm{rp}}{\longrightarrow} H$ and $H$ has a free geometry $F$ as a minor then $G$ also has $F$ as a minor.

PRoof. Express $F$ properly and obtain the corresponding minor $N$ of $G$, then $N \stackrel{\mathrm{rp}}{\longrightarrow} F$. But $N$ cannot have more independent sets than $F$, so $N=F$.

We devote the remainder of this section to discussing the behavior of cir- 
cuits and flats under rp maps. We know from 3.2 that given a map $G \stackrel{\mathrm{rp}}{\longrightarrow} H$ in $P_{X}$ each $G$-circuit contains an $H$-circuit. The next proposition shows that all $H$-circuits are contained in $G$-circuits.

Proposition 5.10. Let $G$ and $H$ be in $P_{X}$. Given a map $G \stackrel{\mathrm{rp}}{\longrightarrow} H$ there exists an injection $i$ from $C_{H}$ to $C_{G}$ having the property that $i(C) \supseteq C$ for all circuits $C$ of $H$ and $r_{H}(i(C))=|i(C)|-1$.

Proof. Let $C$ be a circuit of $H$ with $p \in C$. Extend the independent set $C-p$ to a basis $B$ for $H$. B is also a basis for $G$. Let $i(C)$ be the unique $G$-circuit $p$ forms with $B$. The circuit $i(C)$ contains an $H$-circuit and that $H$-circuit must be the unique circuit that $p$ makes with $B$ in $H$, which is $C$. Thus $i(C) \supseteq$ $C$. The uniqueness of $C$ establishes the injectivity of $i$. We get $r_{H}(i(C))=i(C)$ -1 from the fact that $i(C)-p$ is a subset of the basis $B$ of $H$.

CoROllary 5.11. If there is an $\operatorname{rp}$ map $G \stackrel{\mathrm{rp}}{\longrightarrow} H$, then $\left|C_{G}\right| \geqslant\left|C_{H}\right|$.

Proposition 5.12. Let $G$ and $H$ be in $P_{X}$ with a map $G \stackrel{\mathrm{rp}}{\longrightarrow} H$. There is an injection $j$ from $F_{H}$ to $F_{G}$ having the property that $\mathrm{Cl}_{H}(j(f))=f$ for all flats $f$ of $H$.

Proof. Dualize to obtain the map $G^{*} \stackrel{\mathrm{rp}}{\longrightarrow} H^{*}$ and apply 5.10 to get an injection $i$ from $C_{H^{*}}$ to $C_{G^{*}}$. Recall that hyperplanes of a geometry are exactly the complements of circuits of its dual. Define $j: H_{H} \rightarrow H_{G}$ by $j(h)=X-$ $i(X-h)$. Thus $j(h)$ is the complement of the $G^{*}$ circuit $i(X-h)$ and so is a hyperplane of $G$. Since $i(X-h) \supseteq X-h$ we have $j(h)=X-i(X-h) \subseteq X-$ $(X-h)=h$. Since $h$ is closed in $H$ it follows that $\mathrm{Cl}_{H}(j(h)) \subseteq h$. To get equality we need only show that $r_{H}(j(h)) \geqslant r(H)-1$.

The $G^{*}$-circuit $i(X-h)$ has $H^{*}$-rank one less than its cardinality. Thus we can delete a point $p$ from $i(X-h)$ to get an independent set $i(X-h)-p$ which we extend to a basis $B$ of $H^{*}$. Then $X-B$ is a basis of $H$ whose intersection with $i(X-h)$ contains only the point $p$. Therefore $(X-B)-p$ is an independent set of $H$ of size $r(H)-1$ which is contained in $j(h)$. Thus $r_{H}(j(h))$ $\geqslant r(H)-1$, as required.

We have constructed the injection $j$ on the hyperplanes of $H$. Now apply the same construction to the map $T^{k}(G) \stackrel{\mathrm{rp}}{\longrightarrow} T^{k}(H)$ for $k=1,2, \cdots, r(H)$ to define $j$ for all $H$-flats of rank lower than $r(H)-1$. Define $j(H)=G$ to take care of the one remaining flat.

The injection $j$ can also be viewed as an injection of the lattice of flats $L_{H}$ into $L_{G}$, having the property that it takes flats of rank $r$ to flats of rank $r$. That this injection may not preserve the lattice order is shown in Example 5.13. 
EXAMPLE 5.13. A construction of the injection $j$ of 5.12 for the map $G \stackrel{\text { rp }}{\longrightarrow} H$ given by:

$$
G=\underset{c \bullet \bullet d}{a \bullet \bullet b} \stackrel{\mathrm{rp}}{\longrightarrow} \underset{c}{a \bullet d} b=H .
$$

The respective lattices of flats are:
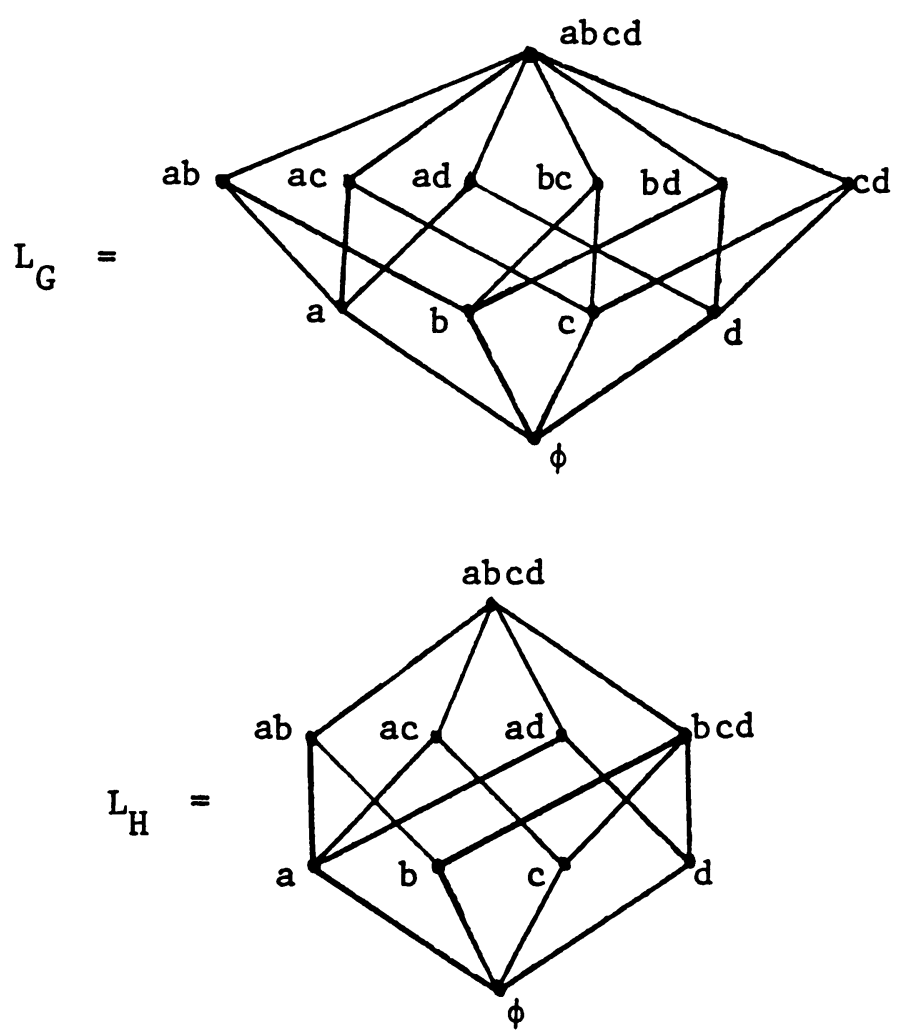

It is clear that $j$ must be the identity map on all $H$-flats other than $b c d$ and that $j$ must take $b c d$ to one of $b c, b d, c d$. No matter which choice is made, however, the map will not preserve order. For example, if $j(b c d)=b c$ then we have $d<b c d$ but $j(d) \not j(b c d)$.

DEFINITION 5.14. Let $W_{r}(G)$ be the number of $G$-flats of $G$-rank $r$. The number $W_{r}(G)$ is called the rth Whitney number of the second kind for the geometry $G$.

COROLlary 5.15. If $G$ and $H$ are in $P_{X}$ with $G \stackrel{\mathrm{IP}}{\longrightarrow} H$ then $W_{r}(G) \geqslant$ $W_{r}(H)$ for all integers $r$. 
Proof. The inequality follows from the injection $j$ of 5.12 .

6. Hereditary properties: Binary geometries.

DEFINTION 6.1. A geometry $G$ in $P_{X, k}$ is said to be coordinatizable over a field $F$ if there exists a $k \times|X|$ matrix $M$ and a bijection $b$ from $X$ to the columns of $M$ such that a subset $I$ of $X$ is independent in $G$ iff the subset $b(I)$ of columns of $M$ is linearly independent over $F$. The matrix $M$ is said to represent $G$ over $F$. If $M$ represents $G$ via the bijection $b$ and $\beta: H \rightarrow G$ is an isomorphism then $M$ represents $H$ via the bijection $b \circ \beta$. We may therefore state unambiguously that $M$ represents the isomorphism class $G \in P_{n}$ provided it represents any representative $G^{\prime}$ of $G$.

Since elementary row operations do not affect the linear dependencies of columns of a matrix, we may represent any coordinatizable geometry $G$ by a matrix $M_{B}$ in row reduced echelon form relative to a given basis $B$ of $G$. We will assume that the first $k$ columns of $M_{B}$ represent the basis $B$ so that $M_{B}$ has the form $M_{B}=[I A]$ where $I$ is the $k \times k$ identity matrix.

In the next proposition we state, without proof, two well-known facts about coordinatizability [16].

PROPOSITION 6.2. For $G$ in $P_{X}$ or $P_{n}$, if $G$ is coordinatizable over a field $F$ then so is $G^{*}$ and so are all minors of $G$.

DEFINITION 6.3. For $G$ in $P_{X}$ or $P_{n}, G$ is called binary if it is coordinatizable over $F_{2}$, the two element field. A matrix representing $G$ over $F_{2}$ is called a binary representation of $G$.

We state without proof some well-known equivalences [16].

PROPOSITION 6.4. For $G$ in $P_{X}$ or $P_{n}$ the following are equivalent:

(a) $G$ is binary.

(b) $G$ does not have $F_{4,2}$, the four point line, as a minor.

(c) The symmetric difference of any two G-circuits is a disjoint union of G-circuits.

We now show that the property of being binary is hereditary under rp maps.

THEOREM 6.5. Let $G$ and $H$ be in $P_{X}$ or $P_{n}$ with a map $G \stackrel{\mathrm{IP}}{\longrightarrow} H$. If $G$ is binary then so is $H$.

Proof. If $H$ is not binary it has $F_{4,2}$ as a minor. Then by $5.9 G$ has $F_{4,2}$ as a minor so that $G$ is not binary.

Theorem 6.5 naturally raises the question of how rp maps affect geometries coordinatizable over fields other than $F_{2}$. Figure 6.6 shows that coordinatizability over fields of characteristic other than two is not preserved by rp maps. 
Figure 6.6. The geometry $G$ on $\{a, b, c, d, e, f, g\}$ whose affine representation is

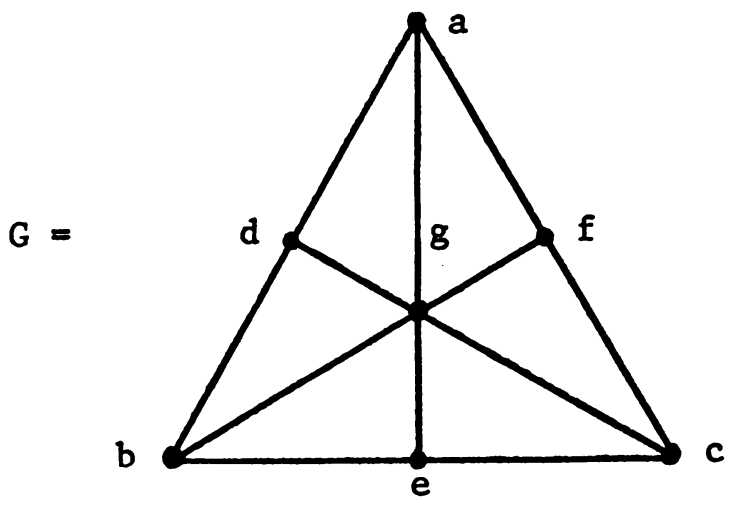

is coordinatizable over any field whose characteristic is not two by the matrix

$$
M=\left[\begin{array}{lllllll}
a & b & c & d & e & f & g \\
1 & 0 & 0 & 1 & 0 & 1 & 1 \\
0 & 1 & 0 & 1 & 1 & 0 & 1 \\
0 & 0 & 1 & 0 & 1 & 1 & 1
\end{array}\right] .
$$

The simple rp image $H$ of $G$ obtained by making the independent set \{def\} of $G$ into a circuit is called the Fano plane and is not coordinatizable over any field of characteristic other than two. The Fano plane is coordinatizable over fields of characteristic two, however. In fact, the matrix $M$, above, represents $H$ over any such field.

Theorem 6.5 shows that the binary geometries (isomorphism classes) form an order ideal within $P_{X, k}\left(P_{n, k}\right)$ under the weak map partial order. The rest of this section will be devoted to studying the properties of this ideal and the nature of the rp maps within it. First we must develop some information concerning the changes in binary representations under rp maps.

Proposition 6.7. Suppose $G$ and $H$ are in $P_{X}$ where $X=\left\{p_{1}, \cdots, p_{n}\right\}$ and suppose that $B=\left\{p_{1}, \cdots, p_{k}\right\}$ is a basis for both $G$ and $H$. Suppose $M_{B}=$ [IA] represents $G$ over $F$ and $M_{B}^{\prime}=\left[I A^{\prime}\right]$ represents $H$ over $F^{\prime}$ where column $c_{j}$ in both matrices corresponds to the point $p_{j}$ of $X$. Then there is a map $G \stackrel{I P}{\longrightarrow} H$ iff whenever a subdeterminant $D$ of $A$ is zero over $F$ then the corresponding subdeterminant $D^{\prime}$ of $A^{\prime}$ (that subdeterminant determined by the same rows and columns) is zero over $F^{\prime}$. 
Proof. Let $G \stackrel{\mathrm{rp}}{\longrightarrow} H$ and suppose the subdeterminant $D$ of $A$ determined by columns $C=\left\{c_{1}^{\prime}, \cdots, c_{m}^{\prime}\right\}$ and rows $R=\left\{r_{1}^{\prime}, \cdots, r_{m}^{\prime}\right\}$ is zero over $F$. Let $B^{\prime}$ be the subset of the first $k$ columns of $M$ (the identity matrix) consisting of those columns which do not have a 1 in the rows of $R$. Then $B^{\prime} \cup C$ is a $k \times k$ submatrix of $M$ with $\operatorname{det}_{M}\left(B^{\prime} \cup C\right)= \pm D=0$. Therefore the subset $S$ of $X$ corresponding to the columns $B^{\prime} \cup C$ is not a basis of $G$. From the rp map, $S$ cannot be a basis of $H$ so the determinant of $B^{\prime} \cup C$ in $M^{\prime}$ must also be zero. So $\operatorname{det}_{M^{\prime}}\left(B^{\prime} \cup C\right)= \pm D^{\prime}=0$, as required.

For the converse, if a $k$-subset of columns $B^{\prime} \cup C$ of $M$ with $B^{\prime} \subset B$ and $C \cap B=\varnothing$ is not a basis of $G$ then $\operatorname{det}_{M}\left(B^{\prime} \cup C\right)= \pm D=0$ where $D$ is the subdeterminant of $A$ determined by the columns of $C$ and the rows of $M$ in which no column of $B^{\prime}$ has a 1 . But then $D^{\prime}$, the corresponding subdeterminant of $M^{\prime}$, is zero. Thus $\operatorname{det}_{M}\left(B^{\prime} \cup C\right)= \pm D^{\prime}=0$ and $B^{\prime} \cup C$ is not a basis for $H$. Therefore any basis of $H$ is a basis of $G$ and we have the map $G \stackrel{\mathrm{rp}}{\longrightarrow} H$.

Corollary 6.8. Let $G$ and $H$ be in $P_{X}$ or $P_{n}$ with $G$ binary and a map $G \stackrel{\mathrm{Ip}}{\longrightarrow} H$. Let $M_{B}=[I A]$ be a binary representation of $H$ for a given basis $B$ of $H$ and let $M_{B}^{\prime}=\left[I A^{\prime}\right]$ be the binary representation of $G$ whose jth column corresponds to the same point $p_{j}$ of $X$ as does the jth column of $M$. If the ijth entry $a_{i j}$ of $M$ is $a 1$ then so is the ijth entry $a_{i j}^{\prime}$ of $M^{\prime}$.

We state without proof the following proposition due to Crapo [5] which will be useful in what follows.

Proposition 6.9. For a connected geometry $G$ in $P_{X}$ and a point $p \in X$ at least one of $G-p$ or $G / p$ is connected.

THEOREM 6.10. Let $G$ and $H$ be in $P_{X}$ or $P_{n}$ with a nontrivial map $G \stackrel{\mathrm{rp}}{\longrightarrow} H$. If $G$ is binary then $H$ is separable.

PRoof. Induction on $n$. If $n=0$ or 1 there is nothing to prove. If $n=2$ the only nontrivial $\mathrm{rp}$ map is $\bullet \stackrel{\mathrm{rp}}{\longrightarrow} \bullet(\bullet)$, for which the theorem holds. Assume the theorem is true for $n<m$ and let $G$ and $H$ have size $m$. If the point $p \in H$ is a loop or isthmus then $H$ is separable and we are done. Otherwise we delete and contract by $p$ and use 5.5 to get the two rp maps: $G-p \stackrel{\mathrm{rp}}{\longrightarrow} H-p$ and $G / p \stackrel{\mathrm{rp}}{\longrightarrow} H / p$.

We first show that these maps cannot both be trivial. Indeed if $G-p=$ $H-p$ and $G / p=H / p$ then $\left|I_{G-p}\right|=\left|I_{H-p}\right|$ and $\left|I_{G / p}\right|=\left|I_{H / p}\right|$. From the fact [1] that $\left|I_{H}\right|=\left|I_{H-p}\right|+\left|I_{H / p}\right|$ it follows that $\left|I_{G}\right|=\left|I_{H}\right|$ and the map $G \stackrel{\mathrm{IP}}{\longrightarrow} H$ is trivial.

There are now three cases to consider. 
(1) $G-p \stackrel{\mathrm{rp}}{\longrightarrow} H-p$ and $G / p \stackrel{\mathrm{rp}}{\longrightarrow} H / p$ are both nontrivial. In this case we have by induction that $H-p$ and $H / p$ are separable so by $6.9 H$ must be separable, as required.

(2) $G-p=H-p$ and $G / p \stackrel{\text { Ip }}{\longrightarrow} H / p$ is nontrivial. Let $M_{B}=[I A]$ be a binary representation of $H$ and $M_{B}^{\prime}=\left[I A^{\prime}\right]$ be the corresponding representation of $G$. Assume that the point $p$ corresponds to column $c_{n}$ of $M$ and $c_{n}^{\prime}$ of $M^{\prime}$. The fact that $G-p=H-p$ means that the first $n-1$ columns of $M$ and $M^{\prime}$ are identical, i.e. $c_{j}=c_{j}^{\prime}$ for all $j<n$. By 6.8 the 1's of $c_{n}$ are also 1's of $c_{n}^{\prime}$. Further, there are some 0 's in $c_{n}$ which are 1's in $c_{n}^{\prime}$ for otherwise we would have $G=H$. Let $R_{0}=$ \{rows $r_{i}$ of $M \mid a_{i n}=1$ \} and let $R_{1}=$ frows $r_{i}$ of $M \mid a_{i n}=0$ but $a_{i n}^{\prime}=1$ \}. Note that $R_{0}$ and $R_{1}$ are both nonempty. Let $L_{1}=$ \{columns $c_{j}$ of $M \mid c_{j}$ has a 1 in a row of $\left.R_{1}\right\}$. Note that $L_{1}$ is nonempty (it contains at least some subset of $\left.\left\{c_{1}, \cdots, c_{k}\right\}\right)$. We will check that no $c_{j} \in L_{1}$ has a 1 in a row of $R_{0}$. If $c_{j}$ has a 1 in the row $r^{\prime}$ of $R_{0}$ and the row $r^{\prime \prime}$ of $R_{1}$ then the $2 \times 2$ submatrix of $A$ determined by columns $c_{j}$ and $c_{n}$ and rows $r^{\prime}$ and $r^{\prime \prime}$ is $\left[\begin{array}{ll}1 & 1 \\ 1 & 1\end{array}\right]$ so it has determinant zero. By 6.7 the corresponding subdeterminant of $A^{\prime}$ must be zero. However that submatrix is $\left[\begin{array}{ll}1 & 1 \\ 1 & 0\end{array}\right]$, a contradiction. Let $R_{2}=$ \{rows $r_{i}$ of $M \mid r_{i} \notin R_{1}$ and $r_{i}$ has a 1 in a column of $L_{1}$ \} and let $L_{2}=$ \{columns $c_{j}$ of $M \mid c_{j} \notin L_{1}$ and $c_{j}$ has a 1 in a row of $R_{2}$ \}. More generally let $R_{l}=$ frows $r_{i}$ of $M \mid r_{i} \notin \bigcup_{i=1}^{l-1} R_{i}$ and $r_{i}$ has a 1 in a column of $L_{l-1}$ \} and let $L_{l}=$ \{columns $c_{j}$ of $M \mid c_{j} \notin \bigcup_{i=1}^{l-1} L_{i}$ and $c_{j}$ has a 1 in a row of $\left.R_{l}\right\}$. Note that if $c_{j} \in L_{l}$ then $c_{j}$ does not have a 1 in any of the columns of $\bigcup_{i=1}^{l-1} L_{i}$. Furthermore no $c^{\prime} \in$ $L_{l}$ has a 1 in a row of $R_{0}$ for, if so, we would have a zero subdeterminant of $A^{\prime}$ which was nonzero in $A$.

Let $L=\bigcup_{l=1}^{\infty} L$ and let $R$ be the set of all rows which have 1's in the columns of $L$, i.e. $R=\bigcup_{l=1}^{\infty} R_{l}$.

Then $L$ is a nonempty set of columns of $M$ all of whose 1's are in the rows of $R$. Let $L^{\prime}$ be the complementary set of columns and let $R^{\prime}$ be the complementary set of rows of $M$. Clearly $c_{n} \in L^{\prime}$.

$R$ was defined so that there are no 1's of columns of $L$ in $R^{\prime}$. There are also no 1's of columns of $L^{\prime}$ in $R$. Indeed if $c$ is a column of $M$ with a 1 in $R$ then let $t$ be the smallest integer such that $c$ has a 1 in a row of $R_{t}$. Then $C$ is in $L_{t}$ and hence not in $L^{\prime}$. It follows that if we alter the matrix $M$ by permuting the rows of $R$ to the top and the columns of $L$ to the left, the resulting matrix will have the form:

$$
\begin{aligned}
& \text { L } \quad L^{\prime} \\
& R^{\prime}\left[\begin{array}{ll}
N_{1} & 0 \\
0 & N_{2}
\end{array}\right] \text {. }
\end{aligned}
$$


Viewed in this form it is clear that no minimal linearly dependent set of columns can contain columns of $L$ and $L^{\prime}$. Therefore, if $X_{1}$ and $X_{2}$ are the subsets of $X$ corresponding to $L$ and $L^{\prime}$ respectively, no circuit of $H$ has nonvoid intersection with both $X_{1}$ and $X_{2}$. Thus the subgeometries of $H$ on the sets $X_{1}$ and $X_{2}$ are separators of $H$. This completes case (2).

(3) $G / p=H / p$ and $G-p \stackrel{\mathrm{rp}}{\longrightarrow} H-p$ is nontrivial. In this case delete and contract $p$ from the dual map $G^{*} \stackrel{\mathrm{rp}}{\longrightarrow} H^{*}$ to get the two maps $(G / p)^{*}=$ $G^{*}-p \stackrel{\mathrm{rp}}{\longrightarrow} H^{*}-p=(H / p)^{*}$ and $(G-p)^{*}=G^{*} / p \stackrel{\mathrm{rp}}{\longrightarrow} H^{*} / p=(H-p)^{*}$, of which the first is trivial. This is precisely the situation of case (2), so applying that proof we find $H^{*}$ is separable. Therefore $H$ is separable and the proof of the theorem is complete.

Corollary 6.11. Let $G$ and $H$ be in $P_{X}$ or $P_{n}$ with a map $G \stackrel{\mathrm{rp}}{\longrightarrow} H$. If $G$ is binary and $M$ is a connected minor of $H$ then $M$ is also a minor of $G$.

Proof. Express $M$ properly as $M=H / A-B$. By 5.8 there is a map $G / A-B \stackrel{\mathrm{rp}}{\longrightarrow} M$. But $G / A-B$ is binary and $M$ is not separable so we must have $G / A-B=M$.

The equivalences stated in the next definition can be found in [16].

Definition 6.12. For $G$ in $P_{X}$ or $P_{n}, G$ is unimodular if it is binary and does not have the Fano plane (see 6.6) or its dual as a minor. Duals and minors of unimodular geometries are unimodular.

$G$ is graphical if it can be represented as a graph. Equivalently, $G$ is graphical if it is unimodular and does not have $K_{5}^{*}$ or $K_{3,3}^{*}$ as minors $\left(K_{5}\right.$ is the complete 5-graph and $K_{3,3}$ is the complete $3 \times 3$ bipartite graph). Minors of graphical geometries are graphical.

$G$ is planar graphical if it can be represented as a planar graph. Equivalently, $G$ is planar graphical if $G$ is graphical and does not contain $K_{5}$ or $K_{3,3}$ as minors.

PROPOSITION 6.13. The properties of being unimodular, graphical, and planar graphical are all preserved by rp maps.

Proof. It suffices to note that the Fano plane, $K_{5}$, and $K_{3,3}$ are connected and to apply 6.11. This proposition is also an immediate consequence of 6.17.

Our next goal is to give a complete description of simple rp maps of binary geometries. Before getting to the main theorems, however, we require a few technical results.

PROPOSITION 6.14. Let $G$ and $H$ be in $P_{X}$ with a map $G \stackrel{\mathrm{IP}}{\longrightarrow} H$. Suppose $F$ is a separator of $H$ and the subgeometry $F^{\prime}$ of $G$ given by $F^{\prime}=G-(X-F)$ 
satisfies $r_{G}\left(F^{\prime}\right)=r_{H}(F)$. Then the map $G \stackrel{\mathrm{rp}}{\longrightarrow} H$ decomposes as follows: $G \stackrel{\mathrm{rp}}{\longrightarrow} G / F^{\prime} \oplus F^{\prime} \stackrel{\mathrm{rp}}{\longrightarrow} H$.

Proof. The map $G \stackrel{\text { Ip }}{\longrightarrow} G / F^{\prime} \oplus F^{\prime}$ exists for any subgeometry $F^{\prime}$ of $G$ since $I_{G / F^{\prime} \oplus F^{\prime}}=\left\{I_{1} \cup I_{2} \mid I_{1} \in I_{G / F^{\prime}}, I_{2} \in I_{F^{\prime}}\right\}=\left\{I_{1} \cup I_{2} \mid I_{1} \cup I_{3} \in I_{G}\right.$ for each maximal independent set $I_{3}$ of $\left.F^{\prime}, I_{2} \in I_{F^{\prime}}\right\} \subseteq I_{G}$. For the map $G / F^{\prime} \oplus F^{\prime} \stackrel{\mathrm{rp}}{\longrightarrow} H$ note that any basis of $H$ is of the form $I_{1} \cup I_{2}$ where $I_{1}$ is a basis for the separator $H-F$ and $I_{2}$ is a basis for $F$. Then $I_{1} \cup I_{2}$ is a basis for $G$ and since $r\left(F^{\prime}\right)=r(F), I_{2}$ is a maximal independent subset of $F^{\prime}$. Therefore $I_{1} \cup I_{2}$ is a basis of $G / F^{\prime} \oplus F^{\prime}$, as required.

LeMma 6.15. Let $G$ be a connected geometry containing more than one point. Either $G$ contains two points either of which can be deleted without separating $G$, or $G^{*}$ contains two points either of which can be deleted without separating $G^{*}$.

Proof. If $G$ has only two points then $G$ must be the two point multiple point $F_{2,1}$. Deleting either point leaves the connected geometry $F_{1,1}$. If $G$ has more than two points let $p_{1}, p_{2}, p_{3}$ be points of $G$. By 6.8 at least one of $G-p_{i}, G / p_{i}$ must be connected for each $i$. Among these three possibilities there must be two connected $G-p_{i}$ 's or two connected $G / p_{i}$ 's and the two $G / p_{i}$ 's provide connected deletions $G^{*}-p_{i}$ of $G^{*}$.

THEOREM 6.16. Let $G$ and $H$ be in $P_{X}$ or $P_{n}$ with $G$ binary and a map $G \stackrel{\mathrm{rp}}{\longrightarrow} H$. There exists some component (minimal separator) $F$ of $H$ such that $F=G-(X-F)$. Otherwise stated, there exists a component $F$ of $G$ which is equal to its preimage in $G$ viewed as a subgeometry of $G$.

Proof. By induction on $n$. If $n=0$ or 1 there is nothing to prove. If $n=2$ the only nontrivial case is $G=a \bullet b \stackrel{\mathrm{Ip}}{\longrightarrow} a \bullet(\bullet b)=H$ and the component $\bullet a$ of $H$ is equal to the subgeometry $\bullet a$ of $G$. Assume the proposition is true for $n<m$ and let $G$ and $H$ have $m$ points. If $H$ is connected then by $6.10 \mathrm{G}=H$ and the proposition is trivially true. Otherwise let $H=F_{1} \oplus F_{2} \oplus$ $\cdots \oplus F_{l}$ where each $F_{i}$ is nonempty and connected. Three cases arise:

(1) Some component $F_{i}$ of $H$ contains only the point $p$. Then $p$ is either an isthmus or a loop of $H$. If $p$ is an isthmus of $H$ then it is certainly equal to the subgeometry $p$ of $G$ and we are done. If $p$ is a loop of $H$, delete $p$ from $H$ and $G$ to get the map $G-p \stackrel{\mathrm{Ip}}{\longrightarrow} H-p$. By induction $H-p$ has a component $F$ such that $F=(G-p)-((X-p)-F)$. But then $F$ is a component of $H$ and $F=G-(X-F)$.

(2) Some component, say $F_{1}$, of $H$ contains three or more points. We apply 6.15 to the component $F_{1}$ and obtain one of two results: (a) There 
exist points $p$ and $q$ of $F_{1}$ such that both $F_{1}-p$ and $F_{1}-q$ are connected. (b) There exist points $p$ and $q$ of $F_{1}^{*}$ such that both $F_{1}^{*}-p$ and $F_{1}^{*}-q$ are connected. If (a) holds, delete $p$ from $H$ and $G$ to get the map $G-p \stackrel{\text { Ip }}{\longrightarrow} H-$ $p=F_{1}-p \oplus F_{2} \oplus \cdots \oplus F_{l}$. By induction some component of $H-p$ is equal to the corresponding subgeometry of $G-p$. If the component in question is $F_{i}$ for some $i \in\{2, \cdots, l\}$ then $F_{i}$ is also a component of $H$ and is equal to the corresponding subgeometry of $G$ so we are done. If none of the $F_{i}$ for $i \in$ $\{2, \cdots, l\}$ is equal to its corresponding subgeometry of $G$, then the component in question must be $F_{1}-p$. Also if none of the $F_{i}$ for $i \in\{2, \cdots, l\}$ will work then the component $F_{1}-q$ of $H-q$ must be equal to its corresponding subgeometry of $G-q$. Letting $F^{\prime}=G-\left(X-F_{1}\right)$ we have $F^{\prime}-p=F_{1}-p$, $F^{\prime}-q=F_{1}-q$, and, obviously, $F^{\prime}-p-q=F_{1}-p-q$. Further, $F_{1}-p$ is connected and has two or more points so $q$ cannot be an isthmus of $F_{1}-p$. Therefore $r_{H}\left(F_{1}-p-q\right)=r_{H}\left(F_{1}-p\right)=r_{H}\left(F_{1}-q\right)$. These same equalities hold in $G$, i.e. $r_{G}\left(F^{\prime}-p-q\right)=r_{G}\left(F^{\prime}-p\right)=r_{G}\left(F^{\prime}-q\right)$, so the rank axioms require that $r_{G}\left(F^{\prime}\right)=r_{G}\left(F^{\prime}-p-q\right)$. In particular $r_{G}\left(F^{\prime}\right)=r_{H}\left(F_{1}\right)$ so we may apply 6.14 to get the decomposition $G \stackrel{\mathrm{IP}}{\longrightarrow} G / F^{\prime} \oplus F^{\prime} \stackrel{\mathrm{IP}}{\longrightarrow} F_{1} \oplus F_{2} \oplus \cdots \oplus F_{l}$. The restriction of the latter map to $F^{\prime} \stackrel{\mathrm{rp}}{\longrightarrow} F_{1}$ has $F^{\prime}$ binary and $F_{1}$ connected so by 6.10 we have $F^{\prime}=F_{1}$, and we are finished with (a). (b) follows from (a) by duality.

(3) Every component $F_{i}$ of $H$ has two points. This means that each $F_{i}$ is a two point multiple point. $H$ is then coordinatized by the binary matrix

$$
M=\left[\begin{array}{c|c}
c_{1}, \cdots, c_{k} & c_{k+1}, \cdots, c_{2 k} \\
I & I
\end{array}\right]
$$

where both $\Gamma$ s represent $k \times k$ identity matrices. By 6.8 we can coordinatize $G$ by a matrix $M^{\prime}$ which consists of adding some 1's to the columns $c_{k+1}$ through $c_{2 k}$ of $M$. If we can show that there is some column $c_{i}^{\prime}$, for $i \in\{k+1, \cdots$, $2 k$ \}, of $M^{\prime}$ to which no 1 was added then the proof is finished, for then the component of $H$ corresponding to columns $c_{i}$ and $c_{i-k}$ of $M$ is still a multiple point of $G$. Therefore suppose that each of the last $k$ columns of $M^{\prime}$ has had at least one 1 added to it. In this case one can check that there is some square submatrix $S^{\prime}$ contained in the submatrix $N$ consisting of the last $k$ columns of $M^{\prime}$ whose main diagonal concides with the main diagonal of $N$ and which has exactly two 1's in each row and column.

Then $S^{\prime}$ has determinant 0 over $F_{2}$ but the corresponding submatrix $S$ of $M$ is the identity, so $\operatorname{det}(S)=1$. By 6.7 , this contradicts the existence of the map $G \stackrel{\text { rp }}{\longrightarrow} H$. 
The proof of Theorem 6.16 is complete.

Theorem 6.16 is not true if $G$ is not binary as shown by the map

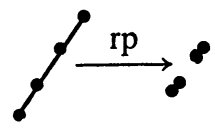

THEOREM 6.17. For $G$ in $P_{X}$ or $P_{n}$ and $G$ binary each simple rp map of $G$ is of the form $G \stackrel{\mathrm{rp}}{\longrightarrow} G / F \oplus F$ for some subgeometry $F$ of $G$.

Proof. First consider the case where $G$ is connected. By 6.10 any simple rp image $H$ of $G$ is separable and by 6.16 there exists a separator $F$ of $H$ with $F=G-(X-F)$. Apply 6.14 to the map $G \stackrel{\mathrm{Ip}}{\longrightarrow} H$ to get the decomposition $G \stackrel{\mathrm{rp}}{\longrightarrow} G / F \oplus F \stackrel{\mathrm{rp}}{\longrightarrow} H$. Since $G$ is connected, the first map is nontrivial so $G / F \oplus F=H$, as required.

If $G$ is separable, the result follows from the fact that $F_{1} \oplus \cdots \oplus F_{i} / F$ $\oplus F \oplus \cdots \oplus F_{m}=\left(F_{1} \oplus \cdots \oplus F_{i} \oplus \cdots \oplus F_{m}\right) / F \oplus F$ for a subgeometry $F$ of $F_{i}$.

Let $\# G$ be the number of components of $G$.

THEOREM 6.18. If $G$ and $H$ are in $P_{X}$ or $P_{n}$ with $G$ binary and there is $a$ simple $\operatorname{rp}$ map $G \stackrel{\mathrm{rp}}{\longrightarrow} H$, then $\# H=\# G+1$.

Proof. A simple map is trivial when restricted to all but one component of a separable geometry, so it suffices to show that if $G$ is connected then $\# H=$ 2. Assume $G$ is connected. By $6.17 H=G / F \oplus F$ for some subgeometry $F$ of $G$. If $G / F$ and $F$ are both connected the proof is over. Otherwise we consider two cases, both of which lead to contradictions.

(1) $F$ is separable. Write $F$ as a direct sum of two nonempty separators, $F=F_{1} \oplus F_{2}$. Then $F_{1}=G-\left(X-F_{1}\right)$ so apply 6.14 to get the decomposition $G \stackrel{\mathrm{rp}}{\longrightarrow} G / F_{1} \oplus F_{1} \stackrel{\mathrm{rp}}{\longrightarrow} G / F \oplus F$. The first map is nontrivial since $G$ is connected. We will show the second map is also nontrivial to contradict the fact that the map $G \stackrel{\mathrm{rp}}{\longrightarrow} H$ is simple.

Since $G$ is connected there exist $G$-circuits with nonempty intersection with $F_{1}$ and $F_{2}$. Let $C$ be such a circuit for which $|C-F|$ is minimal. $|C-F|$ is not zero for then $F_{1}$ and $F_{2}$ could not be separators of $F$. Therefore $C-F$ is a dependent subset of $G / F$. However, $C-F$ is independent in $G / F_{1}$, so the map $G / F_{1} \oplus F_{1} \stackrel{\mathrm{rp}}{\longrightarrow} G / F \oplus F$ is nontrivial. Indeed, if $G-F$ were dependent in $G / F_{1}$ then some subset $S$ of $G-F$ would extend to a circuit $C^{\prime}$ of $G$ such that $C^{\prime}-S \subseteq F_{1}$. Then we find points $q$ in $C \cap F_{2}$ and $p$ in $S$ and apply the circuit axioms to find a $G$-circuit $C^{\prime \prime}$ with $q \in C^{\prime \prime}$ and $C^{\prime \prime} \subseteq C \cup C^{\prime}-p$. Then $C^{\prime \prime}$ intersects both $F_{1}$ and $F_{2}$ and $\left|C^{\prime \prime}-F\right|<|C-F|$, contradicting the minimality of $|C-F|$. 
(2) $G / F$ is separable. Dualize the map $G \stackrel{\mathrm{rp}}{\longrightarrow} G / F \oplus F$ to get $G^{*} \stackrel{\mathrm{rp}}{\longrightarrow}$ $\left(G^{*}-F\right) \oplus G^{*} /(X-F)$. Then the subgeometry $G^{*}-F$ of $G^{*}$ is separable and we can apply the argument of case (1) to obtain the contradiction.

We note in 8.4 that the weak map partial order does not satisfy the Jordan-Dedekind chain condition, even when restricted to $P_{X, k}$ or $P_{n, k}$. We now see that the chain condition does hold within the binary ideal of $P_{X, k}$ and $P_{n, k}$.

Corollary 6.19. Let $G$ be in $P_{X}$ or $P_{n}$ with $G$ binary. Any rp map $G \stackrel{\mathrm{rp}}{\longrightarrow} H$ decomposes into exactly \#H-\#G simple maps.

Proof. By 6.18 each simple map increases the number of components by exactly one.

We have shown that within $P_{X, k}$ or $P_{n, k}$ the connected binary geometries (isomorphism classes in the case of $P_{n, k}$ ) form an antichain in the weak map partial order. The order ideal generated by this antichain contains only binary geometries and it is easy to see (by adding 1's to binary representations) that it contains all the binary geometries of $P_{X, k}$ or $P_{n, k}$ respectively. Within this ideal the chain condition holds, there being $\# H-\# G$ simple rp maps between $G$ and its image $H$. All simple maps within this ideal are of the form $G \stackrel{\mathrm{rp}}{\longrightarrow}$ $G / F \oplus F$ for some connected subgeometry $F$ of $G$.

The connected geometries form an order filter in the weak map partial order on $P_{X, k}$ or $P_{n, k}$ of which the binary geometries are minimal elements. They are not, however, the only minimal elements as is shown by the example of Figure 6.20.

FIGURE 6.20. An affine representation of a connected geometry $G$ (whose dependent hyperplanes are abcde, abcf, abcg, abch, adef, adeg, adeh, afgh, bdfg, $b e g h, c d g h, c e f h)$ which is not binary but for which every simple rp image is separable.

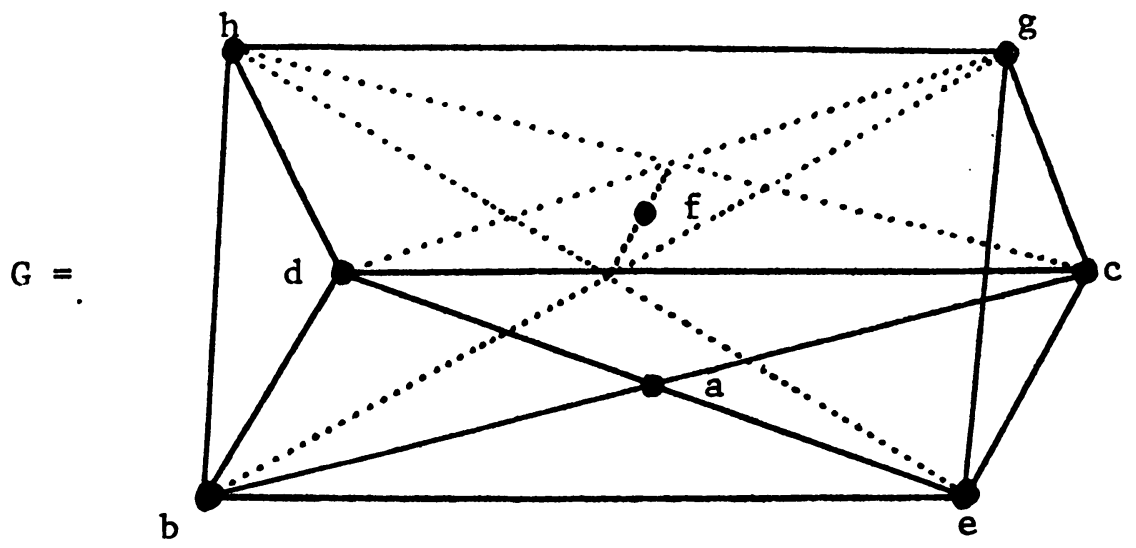


However, $G-f$ is binary. In fact it is representable by the following planar graph:

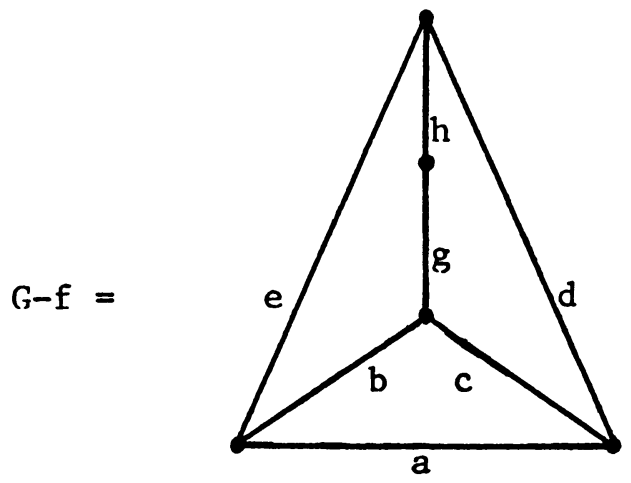

We close this section by proving a proposition which is useful in the theory of bracket rings of geometries [17] where it bears on the question of whether or not the bracket ring of a unimodular geometry is an integral domain.

Proposition 6.21. Let $G$ be a binary geometry in $P_{X, k}$ where $|X|=2 k$. Suppose there is an $\mathrm{rp}$ map $G \stackrel{\mathrm{Ip}}{\longrightarrow} H$. If $B_{1}, X-B_{1}, B_{2}$, and $X-B_{2}$ are all bases of $G$ then $B_{1}$ and $X-B_{1}$ are bases for $H$ iff $B_{2}$ and $X-B_{2}$ are bases for $H$.

Proof. It suffices to prove the proposition when $G \stackrel{\mathrm{rp}}{\longrightarrow} H$ is a simple map. Thus we can assume $H=G / F \oplus F$. Since $B_{1}$ and $X-B_{1}$ are both bases for $G / F \oplus F$ we must have $\left|B_{1} \cap F\right|=\left|\left(X-B_{1}\right) \cap F\right|=r_{G}(F)$, so $|F|=2 r_{G}(F)$. Therefore neither $\left|B_{2} \cap F\right|$ or $\left|\left(X-B_{2}\right) \cap F\right|$ can exceed $r_{G}(F)$ so they both must equal $r_{G}(F)$. Then $B_{2}$ and $X-B_{2}$ are both bases for $G / F \oplus F$.

7. Hereditary properties: Tutte-Grothendieck invariants.

DEFINITION 7.1. An invariant is a function $f$ defined on the class of all geometries such that $f(G)=f(H)$ if $G$ is isomorphic to $H$. It is clear that an invariant $f$ is unambiguously defined for an isomorphism class $G \in P_{n}$ by $f(G)=$ $f\left(G^{\prime}\right)$ for any representative $G^{\prime}$ of $G$. A Tutte-Grothendieck invariant is an invariant taking values in a commutative ring such that $f(G)=f(G / p)+f(G-p)$ for any point $p$ of $G$ which is not an isthmus or a loop and $f(G)=f\left(S_{1}\right) \cdot f\left(S_{2}\right)$ for any complementary separators $S_{1}$ and $S_{2}$ of $G$. The Tutte polynomial, $t_{G}(z, x)$, of $G$ is the unique two variable polynomial function which is a TutteGrothendieck invariant and has the property that $t_{G}(z, x)=z$ if $G$ is an isthmus and $t_{G}(z, x)=x$ if $G$ is a loop. This concept was introduced by Crapo [6] and studied extensively by Brylawski [1]. 
Many interesting invariants can be derived from the Tutte polynomial and its derivatives. The first column of Table 7.2 provides a list of such invariants expressed in terms of $t_{G}(z, x)$ with references given where appropriate. The second column of the table describes the behavior of the invariants under rp maps and refers to the result in this section where the proof is given. Most of the rest of this section is devoted to proving the statements that appear in that second column.

TABLE 7.2. The behavior under rp maps of invariants derived from the Tutte polynomial

$$
\begin{aligned}
& \text { Invariants of } G \text { in terms } \\
& \text { of } t_{G}(z, x)
\end{aligned}
$$

1. $t_{G}(1,1)=\left|B_{G}\right| \quad[1]$

2. $t_{G}(2,1)=\left|I_{G}\right| \quad[1]$

3. $t_{G}(1,2)=\left|I_{G} *\right| \quad[1]$

4. $t_{G}(2,2)={ }_{2}|X| \quad[1]$

5. $t_{G}(0,0)=0 \quad[1]$

6. $t_{G}(1,0)=|\mu(G)|$ where $\mu(G)=$ $\mu(0,1)$ of the lattice $L_{G}$ as defined by Rota [14].

7. $t_{G}(2,0)$, for a graph this invariant is the number of acyclic orientations [15]; for a unimodular geometry it is the number of nonhomogeneous unimodular coordinatizations of $G$ times $2^{\# G}[3]$.

8. $\partial t_{G} /\left.\partial z\right|_{(0,0)}=\beta(G)$ where $\beta(G)$ is the higher invariant described by Crapo [5]

9. $\left(\left(\partial^{k} t_{G} / \partial z^{k}\right) \mid(0,0)\right) / k !=c_{k}$, the coefficient of the term of $t_{G}(z, 0)$ in which $z^{k}$ appears.

10. $t_{G}(1-\lambda, 0)=x_{G}(\lambda)$, the characteristic polynomial of $G[7]$.

11. $\Sigma_{k=l}^{r(G)}\left(l_{l}^{k}\right)\left(\left(\partial^{k} t_{G} / \partial z^{k}\right)|(0,0) / k !=| w_{l} \mid\right.$ where $w_{l}$ is the coefficient of $\lambda^{l}$ in $x_{G}(\lambda)$, also known as the $l$ th Whitney number of the first kind of $G[9]$.
Behavior of these invariants under the nontrivial rp map

$$
G \stackrel{\text { rp }}{\longrightarrow} H \text {. }
$$

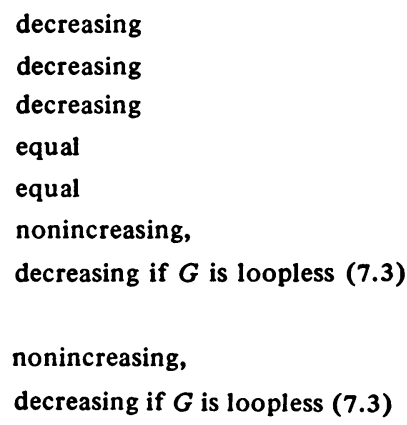

The statements concerning the first five invariants of Table 7.2 require no elaboration. Statements 6 and 7 follow from the next proposition and statements 8,9 , and 11 are a consequence of Proposition 7.4. 
Proposition 7.3. Let $G$ and $H$ be in $P_{X}$ or $P_{n}$ with a nontrivial rp map $G \stackrel{\mathrm{rp}}{\longrightarrow} H$. Then $t_{G}(z, 0) \geqslant t_{H}(z, 0)$ for all $z>0$ and the inequality is strict provided $G$ has no loops.

PROOF. Induction on $n$. If $n<2$ there are no nontrivial rp maps. If $n=2$ the only case is $G=a \boldsymbol{a} b \stackrel{\mathrm{rp}}{\longrightarrow} \boldsymbol{d}(\boldsymbol{b})=H$. In this case $t_{G}(z, 0)=z$ and $t_{H}(z, 0)$ $=0$ and the proposition clearly holds.

Assume the proposition is true for $n<m$ and let $n=m$. If $H$ is a Boolean algebra then it has no nontrival preimages. If $H$ is a pre-Boolean algebra containing a loop then $t_{H}(z, 0)$ is identically zero, whereas $t_{G}(z, 0) \geqslant 0$ for all positive $z$ and $t_{G}(z, 0)>0$ if $G$ has no loops. Otherwise $H$ has a point $p$ which is neither a loop nor an isthmus. From 5.5 we get the maps $G-p \stackrel{\mathrm{IP}}{\longrightarrow} H-p$ and $G / P \stackrel{\mathrm{rp}}{\longrightarrow} H / p$. Recalling that $t_{G}=t_{G-p}+t_{G / p}$ we have by induction that $t_{G-p}(z, 0) \geqslant t_{H-p}(z, 0)$ and $t_{G / p}(z, 0) \geqslant t_{H / p}(z, 0)$, so $t_{G}(z, 0) \geqslant t_{H}(z, 0)$ for all $z>0$. To show that the inequality is strict if $G$ is loopless we need only show that one of the maps $G-p \stackrel{\mathrm{rp}}{\longrightarrow} H-p$ or $G / p \stackrel{\mathrm{rp}}{\longrightarrow} H / p$ is nontrivial and the domain geometry is loopless. $G-p$ is certainly loopless so if $G-p \stackrel{\mathrm{rp}}{\longrightarrow} H-p$ is nontrivial we are done. Therefore assume $G-p=H-p$. Then $G / p \stackrel{\mathrm{rp}}{\longrightarrow} H / p$ is certainly nontrivial for, if not, then $t_{G}=t_{H}$, so $\left|I_{G}\right|=t_{G}(2,1)=t_{H}(2,1)=$ $\left|I_{H}\right|$ and the map $G \stackrel{\text { Ip }}{\longrightarrow} H$ would be trivial. It remains to show that $G / p$ has no loops, or, equivalently, that there is no point $q \in G$ such that $p$ and $q$ form a two point $G$-circuit. Indeed if $p$ and $q$ formed a two point $G$-circuit then either they would form a two point $H$-circuit which, together with the assumption that $G-p=H-p$, would require that $G=H$, or $p$ would be a loop in $H$, contradicting the original assumption on $p$.

Proposition 7.4. If $G$ and $H$ are in $P_{X}$ or $P_{n}$ and there is an rp map $G \stackrel{\mathrm{rp}}{\longrightarrow} H$ then

$$
\left.\frac{\partial^{k} t_{G}}{\partial z^{k}}\right|_{(z, 0)} \geqslant\left.\frac{\partial^{k} t_{H}}{\partial z^{k}}\right|_{(z, 0)}
$$

for all $z \geqslant 0$ and all positive integers $k$.

PROOF. It is trivially true for all geometries of two or fewer points. Assume it is true for all geometries with $n<m$ and let $n=m$. If $H$ is a Boolean algebra there is nothing to prove. If $H$ is a pre-Boolean algebra containing a loop then $\left.\left(\partial^{k} t_{H} / \partial z^{k}\right)\right|_{(z, 0)}$ is identically zero whereas $\left.\left(\partial^{k} t_{G} / \partial z^{k}\right)\right|_{(z, 0)} \geqslant 0$ for all $z \geqslant$ 0 . Otherwise $H$ has a point $p$ which is neither an isthmus nor a loop. Using 5.5 we get the maps $G-p \stackrel{\mathrm{rp}}{\longrightarrow} H-p$ and $G / p \stackrel{\mathrm{rp}}{\longrightarrow} H / p$. By induction these maps satisfy the proposition so the fact that $t_{G}=t_{G-p}+t_{G / p}$ together with the linearity of differentiation gives the desired result. 
The next result follows immediately from the fact [1] that $t_{G}(z, x)=$ $t_{G} *(x, z)$ and 4.3 .

COROllary 7.5. Propositions 7.3 and 7.4 also hold if $z$ is replaced by $x$, evaluations at $(z, 0)$ are replaced by evaluations at $(0, x)$, and loops are replaced by isthmuses.

Evaluations of the characteristic polynomial $\chi_{G}(\lambda)$ of $G$ for values of $\lambda$ greater than 1 play a central role in the theory of combinatorial geometry, governing the critical exponent of a coordinatizable geometry and the minimal number of colors needed for a proper coloring of a graphical geometry [7].

That the behavior of these invariants is not monotone in the weak map partial order is shown in Figure 7.6.

(a) An affine representation of $P_{4,3}$.

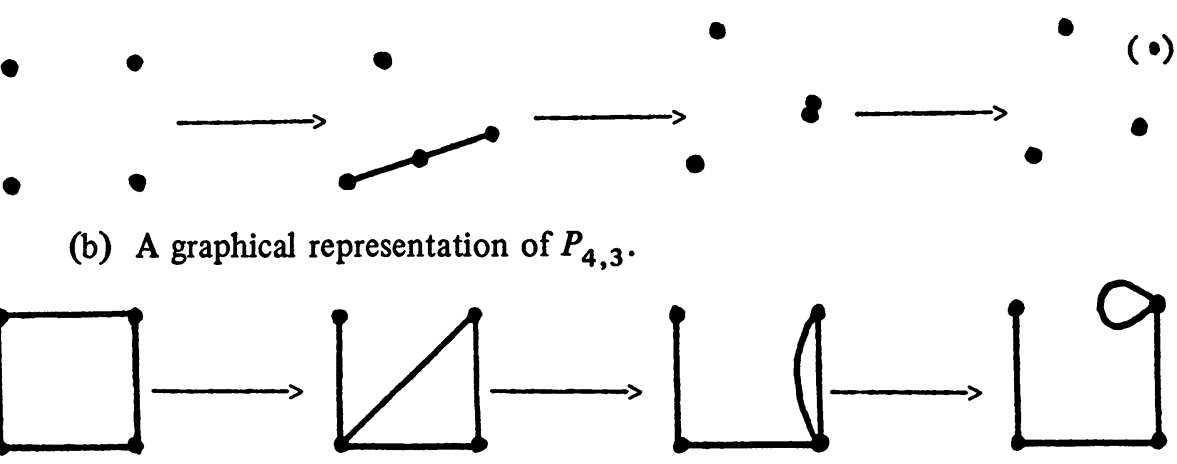

(c) The minimum numbers of colors for a proper coloring.

(d) The critical exponents over $F_{2}$.

FIGURE 7.6. Nonmonotone behavior of important invariants

8. Elementary images and preimages. In this section we discuss various loosely related topics having to do with the relations of strong and weak maps. We begin by introducing a distinguished interval in the weak map partial order.

DEFINTION 8.1. For a geometry $G$ in $P_{X}$, let $\operatorname{Iv}(G)$ represent the interval $[T(G \oplus p), G \oplus(p)]$ in the weak map partial order on $P_{X \cup p} . T(G \oplus p)$ is referred to as $G$ with $p$ added in general position and $G \oplus(p)$ is referred to as $G$ with $p$ added as $a$ loop.

$A$ single point extension of $G$ is a geometry $H$ in $P_{X \cup p}$ such that $r(H)=r(G)$ 
and for which $H-p=G$. It is clear that $T(G \oplus p)$ and $G \oplus(p)$ are single point extensions of $G$.

PROPOSITION 8.2. The interval $\operatorname{Iv}(G)$ consists exactly of the single point extensions of $G$.

Proof. Suppose $H$ is in $\operatorname{Iv}(G)$. Then $T(\check{G} \oplus p) \stackrel{\mathrm{rp}}{\longrightarrow} H \stackrel{\mathrm{rp}}{\longrightarrow} G \oplus(p)$. The point $p$ cannot be an isthmus of $H$ for then it would also be an isthmus of $G \oplus(p)(5.4)$. Thus we can delete $p$ to get the maps $T(G \oplus p)-p \stackrel{\text { Ip }}{\longrightarrow} H-p$ $\stackrel{\text { rp }}{\longrightarrow}(G \oplus(p))-p$. But $T(G \oplus p)-p=(G \oplus(p))-p=G$ so $H-p=G$.

If $H$ is a single point extension of $G$, since $r(H)=r(G)$ we clearly have the map $H \stackrel{\mathrm{rp}}{\longrightarrow} G \oplus(p)$. Further, the map $G \oplus p \rightarrow H$ reduces rank by one so $T(G \oplus p) \stackrel{\mathrm{rp}}{\longrightarrow} H$, as required.

Proposition 8.3. The interval $\operatorname{Iv}(G)$ under the weak map partial order is a lattice in which the supremum of two geometries $F$ and $H$ of $\operatorname{Iv}(G)$ is described by $I_{F \vee H}=I_{F} \cup I_{H}$.

PROof. First let us determine that the set $I_{F \vee H}$ of subsets of $X \cup p$ defined by $I_{F \vee H}=I_{F} \cup I_{H}$ satisfies the independent set axioms. It is clear that $\phi \in I_{F \vee H}$ and subsets of elements of $I_{F \vee H}$ are in $I_{F \vee H}$.

Now suppose $I$ and $J$ are in $I_{F \vee H}$ with $|I|=|J|-1$. We must find some $q \in J$ such that $I \cup q \in I_{F \vee H}$. This condition is clearly met if $I$ and $J$ are both in $I_{F}$ or both in $I_{H}$, so assume that $I \in I_{F}$ and $J \in I_{H}$. Recalling that $I_{F-p}$ $=I_{H-p}=I_{G}$, we see that the only nontrivial case is where both $I$ and $J$ contain $p$ for otherwise they are both in $I_{F}$ or $I_{H}$ or both. Therefore suppose that $p$ is in $I$ and $J$ and look at $\mathrm{Cl}_{F}(I)$, the closure of $I$ in $F$. If there is a point $q \in$ $(J-p)-\mathrm{Cl}_{F}(I)$ then $I \cup q$ is in $I_{F}$ and we are done. Otherwise $(J-p) \subseteq$ $\mathrm{Cl}_{F}(I)$. But $J-p$ is independent in $F$ and $|J-p|=|I|=r\left(\mathrm{Cl}_{F}(I)\right)$ so that $\mathrm{Cl}_{F}(J-p)=\mathrm{Cl}_{F}(I) \supset I$. But $\mathrm{Cl}_{F}(J-p)-p=\mathrm{Cl}_{G}(J-p)=\mathrm{Cl}_{H}(J-p)-p=$ $\mathrm{Cl}_{H}(J-p)$, the latter equality since $J$ is independent in $H$. Therefore $I-p \subseteq$ $\mathrm{Cl}_{H}(J-p)$. But $|I-p|=|J-p|-1$ so $\mathrm{Cl}_{H}(I-p) \varsubsetneqq \mathrm{Cl}_{H}(J-p)$. Therefore there exists a point $q \in J-p$ such that $(I-p) \cup q$ is independent in $H$ and $\mathrm{Cl}_{H}((I-p) \cup q)=\mathrm{Cl}_{H}(J-p)$. But then $p \notin \mathrm{Cl}_{H}((I-p) \cup q)$ so $(I-p) \cup$ $q \cup p=I \cup q$ is independent in $H$. We have established that $F \vee H$ is a geometry.

$F \cup H$ is clearly a supremum since any geometry $E$ with $E \stackrel{\mathrm{rp}}{\longrightarrow} F$ and $E \stackrel{\mathrm{rp}}{\longrightarrow} H$ must have $I_{E} \supseteq I_{F} \cup I_{H}=I_{F \vee H}$. Finally $F \vee H$ is in $\operatorname{Iv}(G)$ since

$$
I_{(F \vee H)-p}=\left\{I \in I_{F} \cup I_{H} \mid p \notin I\right\}=I_{F-p} \cup I_{H-p}=I_{G} \text {. }
$$

That $\operatorname{Iv}(G)$ does not satisfy the Jordan-Dedekind chain condition is shown in Figure 8.4. 
FIGURE 8.4. An affine representation of $\operatorname{Iv}(G)$ where $G$ is represented by

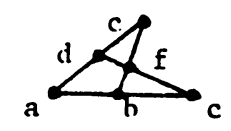

Only the position of $p$ is indicated. Note that the chain condition is not satisfied.

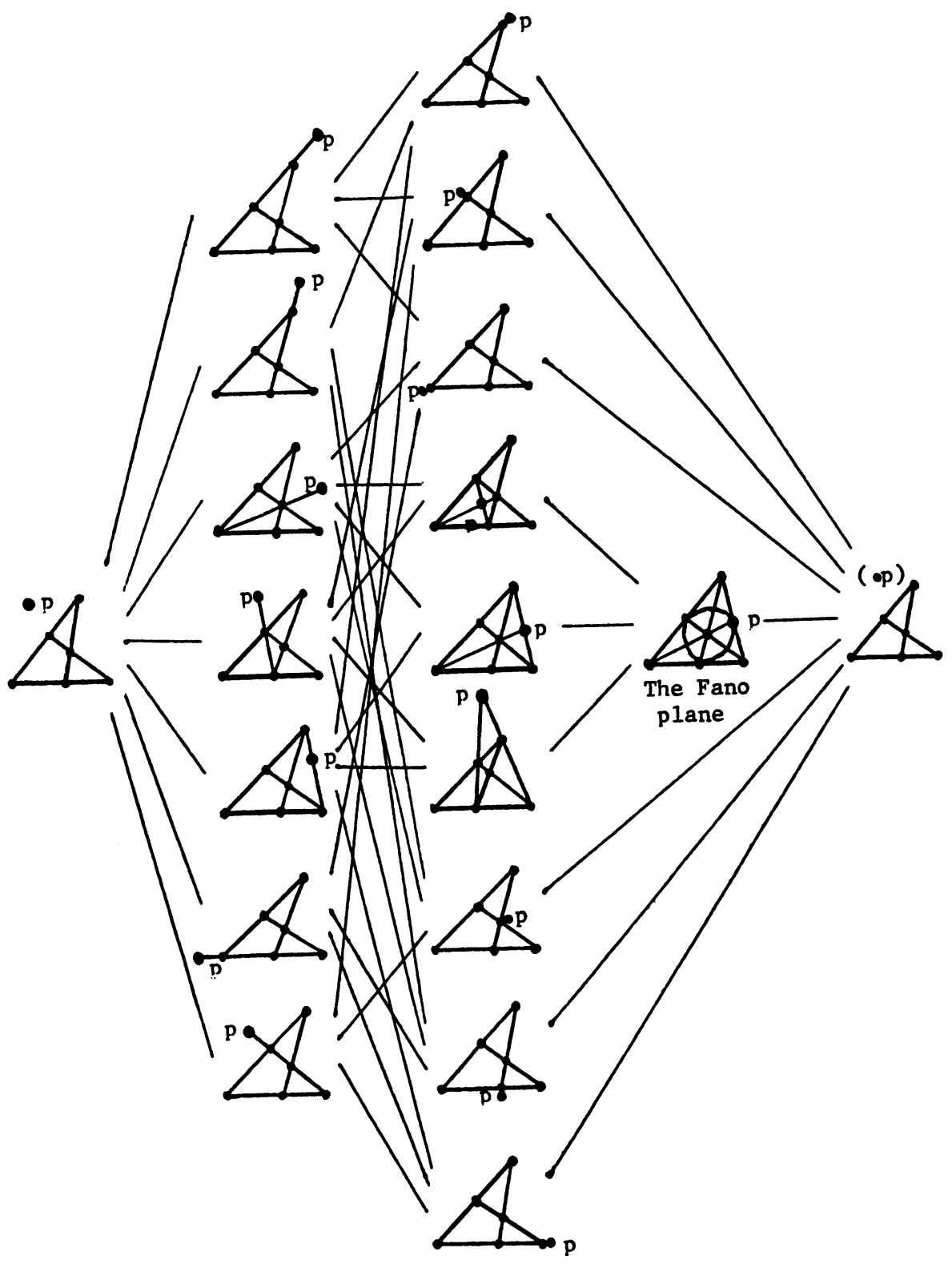


The lattice $\operatorname{Iv}(G)$ is closely related to some more familar structures in a manner which we describe below.

DEfinition 8.5. Suppose $G$ is in $P_{Z}$ and $L_{G}$ is the lattice of flats of $G$. The pair $(X, Y)$ of flats in $L_{G}$ is called a modular pair if $r_{G}(X)+r_{G}(Y)=$ $r_{G}(X \vee Y)+r_{G}(X \wedge Y)$. A modular cut of the lattice $L_{G}$ is a nonempty order filter $M$ in $L_{G}$ having the property that if $X$ and $Y$ are in $M$ and $(X, Y)$ is a modular pair then $X \wedge Y$ is in $M$. There is a 1-1 correspondence between single element extensions of $G$ and modular cuts of $L_{G}$ [4], given as follows: If $H$ is a single point extension of $G$ then the set of all $G$-flats $f$ such that $r_{G}(f)=$ $r_{H}(f \cup p)$ is a modular filter of $L_{G}$ and all modular filters determine single element extensions in this manner.

Higgs [11] and Brylawski [1] have shown that for each elementary map $G \stackrel{\text { st }}{\longrightarrow} H$ where $G$ and $H$ are in $P_{X}$ there exists a unique geometry $F$ on the set $X \cup p$ such that $G=F-p$ and $H=F / p$. Equivalently, there is a $1-1$ correspondence between single element extensions of a geometry $G$ (other than $G \oplus$ $(p)$ ) and elementary images of $G$ which sends each single element extension $F$ to the elementary image $F / p$. By dualizing one sees that there is a 1-1 correspondence between single element extensions of $G^{*}$ and elementary preimages of $G$ which sends each single element extension $F$ of $G^{*}$ to the elementary preimage $F^{*}-p$ of $G$. Shortly, we will relate all these notions to the lattice $\operatorname{Iv}(G)$.

First we prove a general proposition about extending maps.

Proposition 8.6. Let $G$ and $H$ be in $P_{X \cup p}$ with $p$ not an isthmus of $G$ or $H$ and suppose there is a map $G-p \stackrel{\mathrm{rp}}{\longrightarrow} H-p$. Let $M_{G-p}$ be the modular cut of $L_{G-p}$ consisting of all $G-p$ flats $f$ such that $r_{G-p}(f)=r_{G}(f \cup p)$. There is a map $G \stackrel{\mathrm{rp}}{\longrightarrow} H$ iff $\left\{\mathrm{Cl}_{H-p}(f) \mid f \in M_{G-p}\right.$ and $\left.r_{G-p}(f)=r_{H-p}(f)\right\} \subseteq$ $M_{H-p}$.

Proof. Suppose $G \stackrel{\mathrm{Ip}}{\longrightarrow} H$ and let $f$ be in $M_{G-p}$ with $r_{G-p}(f)=r_{H-p}(f)$. Let $I$ be a maximal $H$-independent subset of $f$. Then $I$ is also a maximal $G$-independent subset of $f$. Since $f \in M_{G-p}$ we have that $r_{G-p}(f)=r_{G}(f \cup p)$ so $p$ makes a $G$-circuit $C$ with $I$. This circuit contains a circuit $C^{\prime}$ in $H$ and $C^{\prime}$ must contain $p$. Therefore $p \in \mathrm{Cl}_{H}(I)=\mathrm{Cl}_{H}(f)$. So $r_{H-p}\left(\mathrm{Cl}_{H-p}(f)\right)=|I|=$ $r_{H}\left(\mathrm{Cl}_{H-p}(f) \cup p\right)$ and $\mathrm{Cl}_{H-p}(f) \in M_{H-p}$.

Conversely, suppose the latter condition holds. Since $r(G)=r(H)$ and we have the map $G-p \stackrel{\mathrm{rp}}{\longrightarrow} H-p$ we need only show that independent sets of $H$ containing $p$ are independent in $G$. Let $I$ be independent in $H$ with $p \in I$. Then $\mathrm{Cl}_{H-p}(I-p) \notin M_{H-p}$. But $r_{G-p}\left(\mathrm{Cl}_{G-p}(I-p)\right)=|I-p|=r_{H-p}\left(\mathrm{Cl}_{G-p}(I-p)\right)$ so $\mathrm{Cl}_{G-p}(I-p) \notin M_{G-p}$. Thus 


$$
r_{G-p}(I-p)=r_{G-p}\left(\mathrm{Cl}_{G-p}(I-p)\right)<r_{G}\left(\mathrm{Cl}_{G-p}(I-p) \cup p\right)=r_{G}(I)
$$

so $I$ is independent in $G$.

COROllary 8.7. The 1-1 correspondence between single element extensions and modular cuts of $G$ which was described in 8.5 is a lattice isomorphism from $\operatorname{Iv}(G)$ to the lattice of modular cuts ordered by containment.

For the purposes of the next proposition let us define a 0-less lattice to be a partially ordered set that lacks only a least element to be a lattice. The first part of this proposition appeared in [8].

Proposition 8.8. Suppose $G$ is an $P_{X, k}$. The elementary images and preimages of $G$ form 0-less lattices under the weak map partial order on $P_{X, k-1}$ and $P_{X, k+1}$ respectively. The former 0 -less lattice is isomorphic with $\operatorname{Iv}(G)$ with its least element removed and is obtained by contracting each member of $\operatorname{Iv}(G)$ (except $G \oplus(p))$ by $p$. The latter is isomorphic with $\operatorname{Iv}\left(G^{*}\right)$ with its least element removed.

Proof. It was stated in 8.5 that the function sending the single element extension $F$ of $G(F \neq G \oplus(p))$ to the elementary image $F / p$ was a bijection. If $F^{\prime}$ and $F^{\prime \prime}$ are in $\operatorname{Iv}(G)-\{G \oplus(p)\}$ and there is a map $F^{\prime} \stackrel{\mathrm{rp}}{\longrightarrow} F^{\prime \prime}$ then we have $F^{\prime} / p \stackrel{\mathrm{rp}}{\longrightarrow} F^{\prime \prime} / p$ since $p$ is not a loop of $F^{\prime \prime}$. Conversely if $F^{\prime} / p \stackrel{\mathrm{rp}}{\longrightarrow} F^{\prime \prime} / p$ then the independent sets of $F^{\prime \prime}$ containing $p$ must be independent in $F^{\prime}$ and the independent sets of $F^{\prime \prime}$ not containing $p$ are just the independent sets of $G$. Therefore $F^{\prime} \stackrel{\mathrm{rp}}{\longrightarrow} F^{\prime \prime}$, as required.

The statement concerning elementary preimages now follows from the observation that the elementary preimages of $G$ are exactly the duals of the elementary images of $G^{*}$. The images of a geometry $G$ do not, in general, form a convex set in the weak map partial order. For instance
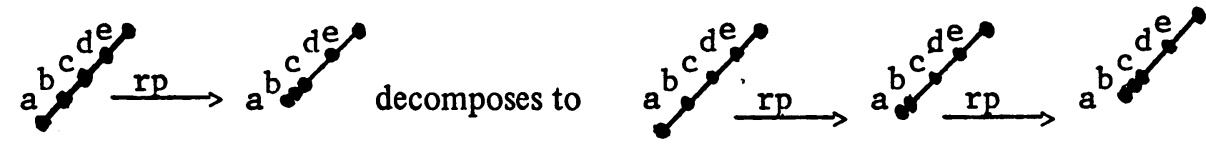

but the former and latter geometries are elementary images of

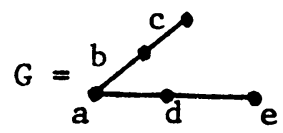

while the middle geometry is not. 
Before leaving the topic of extensions of maps let us make a few further comments concerning the existence and nonexistence of certain extensions.

Proposition 8.9. Let $G$ be in $P_{X \cup p}$ with $p$ not an isthmus of $G$ and suppose there is a map $G-p \stackrel{\mathrm{rp}}{\longrightarrow} H$. The set $E$ of all extensions $H^{\prime}$ of $H$ for which there is a map $G \stackrel{\mathrm{rp}}{\longrightarrow} H^{\prime}$ is a nonempty lattice ideal of $\operatorname{Iv}(H)$. The largest element of $E$ is thus the unique freest extension of the map $G-p \stackrel{\text { IP }}{\longrightarrow} H$ to a map on $G$.

Proof. $E$ is nonempty since it clearly contains $H \oplus(p)$. For the rest it suffices to show that $E$ is closed under suprema. Suppose $H_{1}$ and $H_{2}$ are in $E$. Then $I_{H_{1}} \subseteq I_{G}$ and $I_{H_{2}} \subseteq I_{G}$. Therefore $I_{H_{1}} \cup I_{H_{2}}=I_{H_{1}} \vee_{H_{2}} \subseteq I_{G}$ and $H_{1} \vee H_{2}$ is an image of $G$.

The analogous statement for extensions of preimages $F \stackrel{\mathrm{Ip}}{\longrightarrow} G-p$ is not true. The best that can be said is that the set of all extensions $F^{\prime}$ of $F$ such that $F^{\prime} \stackrel{\mathrm{Ip}}{\longrightarrow} G$ is a nonempty order filter of $\operatorname{Iv}(F)$. That it is not closed under infima is shown by the following example: Let

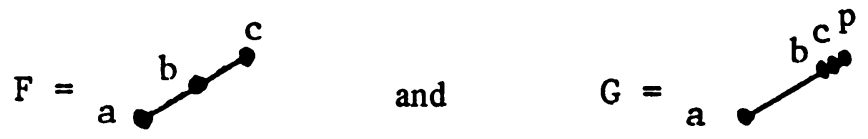

Then there is a map $F \stackrel{\mathrm{rp}}{\longrightarrow} G-p$. Letting

$$
F_{1}=a \text { and } F_{2}=a
$$

it is clear that $F_{1}$ and $F_{2}$ are in $\operatorname{Iv}(F)$ and there are maps $F_{i} \stackrel{\mathrm{rp}}{\longrightarrow} G$. However

$$
F_{1} \wedge F_{2}=a \rho^{c}(\bullet p)=F \oplus(p)
$$

so there is no map from $F_{1} \wedge F_{2}$ to $G$.

There is one potentially useful situation in which extensions may not exist. Suppose we have a map $F \stackrel{\mathrm{rp}}{\longrightarrow} H$ and suppose that the deletion map decomposes to $F-p \stackrel{\mathrm{rp}}{\longrightarrow} G \stackrel{\mathrm{rp}}{\longrightarrow} H-p$. The following example shows that there may exist no extension $G^{\prime}$ of $G$ for which there are maps $F \stackrel{r p}{\longrightarrow} G^{\prime} \stackrel{r p}{\longrightarrow} H$. After deleting $p$ the map

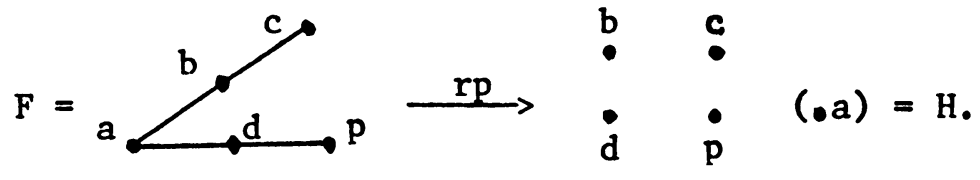


decomposes to

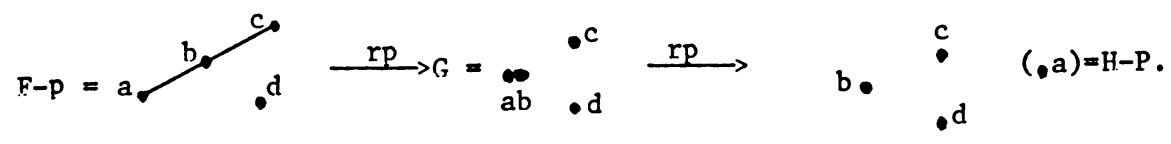

However there is no extension $G^{\prime}$ of $G$ for which there are maps $F \stackrel{\text { Pp }}{\longrightarrow} G^{\prime}$ $\stackrel{\mathrm{rP}}{\longrightarrow} H$.

In [10] Higgs described a lattice order on $P_{X}$ as follows: associate with each $G$ in $P_{X}$ the vector $V_{G}=\left(R_{0}(G), R_{1}(G), \cdots, R_{n}(G)\right)$ where $R_{i}(G)=$ $\left\{S \subseteq X \mid r_{G}(S)=i\right\}$. These vectors are ordered by $V_{G} \geqslant V_{H}$ if there is some integer $k$ such that $R_{i}(G)=R_{i}(H)$ for $i=0,1,2, \cdots, k-1$ and $R_{k}(G) \varsubsetneqq R_{k}(H)$. It is easy to see that the induced order on $P_{X}$ is a lattice with greatest element $B_{X}$ and smallest element $B_{X}^{*}$.

The Higgs lattice order and the weak map partial order are related by the following proposition.

PROPOSITION 8.10. The identity map from $P_{X}$ under the weak map partial order to $P_{X}$ under the Higgs lattice order is an order preserving map.

Proof. Let $G$ and $H$ be in $P_{X}$ and suppose there is a map $G \rightarrow H$. We wish to compare the vectors $V_{G}$ and $V_{H}$. Suppose $k$ is the smallest integer for which $R_{k}(G) \neq R_{k}(H)$. If the subset $S$ of $X$ is in $R_{k}(G)$, then $r_{G}(S)=k$. Thus the map $G \rightarrow H$ requires that $r_{H}(S) \leqslant k$. But we cannot have $r_{H}(S)=l<k$ for then $R_{l}(G) \neq R_{l}(H)$. Therefore $r_{H}(S)=k$ so $R_{k}(G) \varsubsetneqq R_{k}(H)$ and we are done.

9. Final remarks. This section is a brief discussion of a remaining problem in the theory of weak maps.

The fundamental problem is to find a complete description of the action of simple rp maps on geometries. The importance of this problem lies in the fact that any geometry $G$ can be constructed from the free geometry of appropriate size and rank by the action of a sequence of simple rp maps.

For instance, we have seen in 7.2 and 5.15 that the Whitney numbers of the first and second kinds behave systematically under rp maps. The question of whether a geometry exists for which the Whitney numbers of either kind are not unimodal is equivalent to the question of whether such a geometry can be constructed by a single simple rp map from a geometry in which the Whitney numbers are unimodal. A complete understanding of simple rp maps might resolve this question.

In $\S 6$ we described simple rp maps of binary geometries. That description does not hold for arbitrary maps. We conjecture, however, that it holds in the following case. 
Conjecture 9.1. If $G$ and $H$ are in $P_{X}$ or $P_{n}$ with \#G<\#H and if there is a simple map $G \stackrel{\mathrm{rp}}{\longrightarrow} H$ then $H=G / F \oplus F$ for some subgeometry $F$ of G. Further, $\# H=\# G+1$.

\section{REFERENCES}

1. T. H. Brylawski, A decomposition for combinatorial geometries, Trans. Amer. Math. Soc. 171 (1972), 235-282. MR 46 \#8869.

2. An outline for the study of combinatorial pregeometries, Notes, Univ. of North Carolina, 1972.

3. T. H. Brylawski and D. Lucas, Uniquely representable combinatorial geometries, Proc. Internat. Colloq. Combinatorial Theory (Rome, Italy, 1973) (to appear).

4. H. H. Crapo, Single-element extensions of matroids, J. Res. Nat. Bur. Standards Sect. B 69 (1965), 55-65. MR 32 \#7461.

5. - A higher invariant for matroids, J. Combinatorial Theory 2 (1967), 406-417. MR 35 \#6579. \#6705.

6. - The Tutte polynomial, Aequationes Math. 3 (1969), 211-229. MR 41

7. H. H. Crapo and G.-C. Rota, Combinatorial geometries (preliminary ed.), M. I. T. Press, Cambridge, Mass., 1970.

8. T. A. Dowling and D. G. Kelly, Elementary strong maps between combinatorial geometries, Proc. Internat. Colloq. Combinatorial Theory (Rome, Italy, 1973) (to appear).

9. T. A. Dowling and R. M. Wilson, The slimmest geometric lattice (to appear).

10. D. A. Higgs, $A$ lattice order on the set of all matroids on a set, Canad. Math. Bull. 9 (1966), 684-685.

11. - Strong maps of geometries, J. Combinatorial Theory 5 (1968), 185191. MR 38 \#89.

12. D. Kennedy, Factorizations and majors of geometric strong maps, Ph.D. Thesis, Unive of North Carolina, 1973.

13. D. Lucas, Properties of rank preserving weak maps, Bull. Amer. Math. Soc. 80 (1974), 127-131.

14. G.-C. Rota, On the foundations of combinatorial theory. I: Theory of Möbius functions, Z. Wahrscheinlichkeitstheorie und Verw. Gebiete 2 (1964), 340-368. MR 30 \#4688.

15. R. P. Stanley, Acyclic orientations of graphs (to appear).

16. W. T. Tutte, Lectures on matroids, J. Res. Nat. Bur. Standards Sect. B 69 (1965), 1-47. MR 31 \#4023.

17. N. White, The bracket ring and combinatorial geometry, Ph.D. Thesis, Harvard University, 1971.

\section{DEPARTMENT OF MATHEMATICS, UNIVERSITY OF NORTH CAROLINA, CHAPEL HILL, NORTH CAROLINA 27514}

Current address: Ketron, Inc., 1400 Wilson Boulevard, Arlington, Virginia 22209 